\title{
Parametric study on thermal and hydraulic characteristics of laminar flow in microchannel heat sink with fan-shaped ribs on sidewalls - Part 1: heat transfer
}

Lei Chai ${ }^{\mathrm{a},}$, Guo Dong Xia ${ }^{\mathrm{b}}$, Hua Sheng Wang ${ }^{\mathrm{a}}$

${ }^{\text {a }}$ School of Engineering and Materials Science, Queen Mary University of London, Mile End Road, London E1 4NS, UK

${ }^{\mathrm{b}}$ Key Laboratory of Enhanced Heat Transfer and Energy Conservation, Ministry of Education, College of Environmental and Energy Engineering, Beijing University of Technology, Beijing, China

\begin{abstract}
In this first part of a three-part study, a three-dimensional numerical model has been conducted to study the heat transfer characteristics of laminar flow in microchannel heat sinks with different geometric fan-shaped ribs on sidewalls for Reynolds number ranging from 187 to 715 . The numerical model considers entrance effect, conjugate heat transfer, viscous heating and temperature-dependent thermo-physical properties. The water and silicon are used as fluid and solid for the computational domain. The computations base on the finite volume method and implement the SIMPLEC algorithm. The microchannel has the width of $0.1 \mathrm{~mm}$ and depth of $0.2 \mathrm{~mm}$ in the constant cross-section region. The geometric parameters include the width $(0.05-0.4 \mathrm{~mm})$, height $(0.005-0.025 \mathrm{~mm})$ and spacing $(0.2-5 \mathrm{~mm})$ of aligned or offset fan-shaped ribs. The present study firstly examines the effect of fan-shaped ribs on velocity contour, temperature distribution and local heat transfer characteristics for laminar flow in such microchannel heat sinks. It is apparent that the fan-shaped ribs can effectively
\end{abstract}

\footnotetext{
${ }^{*}$ Corresponding author. Tel.: +44 2078827306.

E-mail address: 1.chai@qmul.ac.uk (Lei Chai).
} 
prevent the decline of local heat transfer coefficient along the flow direction. Secondly, the average Nusselt number and the total thermal resistance for such microchannel heat sinks have been studied. Results show that the height and spacing of fan-shaped ribs have significant influences on the heat transfer characteristics, while the width of ribs shows less. For the microchannel heat sinks with lower height and lager spacing of fan-shaped ribs, the aligned arrangement shows higher heat transfer coefficient than the offset one. For the studied Reynolds number range and geometric parameters of fan-shaped ribs, there are a 6-101\% increase in average Nusselt number and a 3-40\% decrease in total thermal resistance for the microchannel heat sink with aligned fan-shaped ribs, while a $4-103 \%$ increase in average Nusselt number and a $2-42 \%$ decrease in total thermal resistance for the microchannel heat sink with offset fan-shaped ribs, comparing with the smooth one. New heat transfer correlations for such microchannel heat sinks are developed, which fit the present data with a mean absolute error of $2.5 \%$ for the ones with aligned fan-shaped ribs and $3.8 \%$ for the ones with aligned fan-shaped ribs.

Key words microchannel heat sink; fan-shaped ribs; heat transfer; thermal resistance

\section{Introduction}

Since the pioneering work by Tuckerman and Pease [1] in the early 1980s, microchannel heat sink incorporating single-phase liquid flow has been widely used in a variety of high heat flux applications as one of the most promising high efficiency heat exchange technologies. More recently, with the rapid development of ULSIC (Ultra-Large-Scale-Integrated-Circuit), 
the chip-level heat flux has been tremendously increased largely due to the increase in processing speed and demand for miniaturization, and several more efficient cooling methods are developed to keep the device at its allowable temperature. Among these methods, microchannel heat sink with passive microstructures is considered to be efficient means to meet this demand. Many efforts have been made for the research of thermal and frictional characteristics in such microchannel heat sinks.

Xu et al. [2,3] experimentally and numerically studied heat transfer with laminar flow in a microchannel heat sink comprising parallel longitudinal microchannels with several transverse microchambers. They found that the redeveloping flow was repeated for all of the independent zones and the repeated thermal developing flow greatly enhanced the overall heat transfer. Subsequently, Chai et al. [4] introduced the staggered rectangular ribs into the transverse microchambers and studied the heat transfer characteristics in such heat sinks. The geometry parameters (e.g. the length and width of rib) and position parameters (e.g. the distance from the parallel microchannels to the rib row and the space between two adjoining transverse microchambers) were investigated. They found that the staggered rectangular ribs could further improve the heat transfer performance largely due to better flow separation and mixing of hot and cold liquids.

Cheng [5] numerically simulated heat transfer in a stacked two-layer microchannel heat sink with passive microstructures. The important parameters (e.g. the ratio of embedded structure height to microchannel height and fluid property) were investigated. They found that the enhanced mixing mechanism of cold and hot fluids caused by the passive microstructures could lead to better heat transfer and lower thermal resistance. The increase of the ratio of the 
height of passive microstructure to microchannel height led to lower thermal resistance. Promvonge et al. [6] carried out a numerical investigation to examine laminar flow and heat transfer characteristics in a three-dimensional isothermal wall square channel with $45^{\circ}$-angled baffles. To generate a pair of mainstream wise vortex flows through the tested section, baffles with an attack angle of $45^{\circ}$ were mounted in tandem for both inline and staggered arrangements on the lower and upper walls of the channel. They found that in each of the main vortex flows, a pair of stream wise twisted vortex (P-vortex) flows created by the $45^{\circ}$ baffle existed and helped to induce impinging flows on a sidewall and wall of the baffle cavity, which resulted in drastic increase in heat transfer rate over the smooth channel. Liu et al. [7] experimentally investigated the fluid flow and heat transfer characteristics in microchannel heat sink with longitudinal vortex generators (LVGs) for Reynolds numbers of 170-1200 with hydraulic diameter of $187.5 \mu \mathrm{m}$ and aspect ratio of 0.067 for LVGs with different number of pairs and angles of attack. Subsequently, Ebrahimi et al. [8] numerically investigated the liquid flow and conjugated heat transfer performance of single-phase laminar flow in such microchannel heat sinks. Five different configurations of the microchannel with different angles of attack of the LVGs were considered. Their results showed that for Reynolds number ranging from 100 to 1100 , there was a $2-25 \%$ increase in the Nusselt number for microchannels with LVGs.

Chai et al. $[9,10]$ numerically investigated the fluid flow and heat transfer characteristics in microchannel heat sinks with fan-shaped reentrant cavities in sidewall and analyzed the effects of structural parameters in such microchannel heat sinks. The structural parameters included the lengths and widths of the constant cross-section region and the reentrant cavity 
region. They attributed the heat transfer enhancement to the periodic thermal developing flow, the increased heat transfer surface area and the jet and throttling effects caused by the changed flow passage. Subsequently, Xia et al. [11] numerically investigated the effect of geometric parameters on water flow and heat transfer characteristics in microchannel heat sink with triangular reentrant cavities. They designed four variables, representing the spacing and geometry of the triangular reentrant cavity to analyze their effects on fluid flow and heat transfer. They found that the vortices in the triangular reentrant cavities led to chaotic advection and could greatly enhance the convective fluid mixing. The thermal and hydraulic boundary layers were interrupted and the repeated developing flow enhanced heat transfer in the constant cross-section region. Chai et al. [12] experimentally and numerically studied the heat transfer characteristics of microchannel heat sinks with periodic expansion-constriction cross-sections. Each heat sink consisted of 10 parallel microchannels with $0.1 \mathrm{~mm}$ wide and $0.2 \mathrm{~mm}$ deep in constant cross-section region and each microchannel consisted of an array of periodic expansion-constriction cross-sections (fan-shape and triangular reentrant cavities). Their results indicated that such microchannel heat sinks could significantly improve heat transfer performance due to the interruption of boundary layer formation, establishment of secondary flow and provision of more surface area.

Foong et al. [13] numerically studied the fluid flow and heat transfer characteristics of a square microchannel with four longitudinal internal fins. Their analysis was carried out for different fin heights and flow parameters. They concluded that the following beneficial features were realized in a microchannel with internal longitudinal fins as compared to an unfinned microchannel: shorter length required for the development of the thermal boundary 
layer, better flow mixing, steeper velocity gradient at the heated surface, increase in the surface area for heat transfer, and increase in surface heat transfer coefficient and Nusselt number. Xie et al. [14] numerically investigated thermal and fluid flow characteristics of microchannel heat sinks with internal vertical Y-shaped bifurcations. The effect of the length of Y-shaped bifurcation and the angles of the arms on the overall performance were examined. They found that the thermal performance of such microchannel heat sinks was much better than the traditional rectangular microchannel. The longest internal Y-shaped bifurcation microchannel resulted in best thermal performance and a larger arm angle led to a better thermal performance for the internal Y-shaped bifurcation microchannel.

Based on the passive heat transfer enhancement methods mentioned above, Chai et al. [15] introduces the offset ribs to the two opposite channel sidewalls for rectangular microchannel heat sink, with the purposes to provide shorter length required for repeated thermal developing flow, to work as micromixers or baffles for better mixing of cold and hot fluids, to be easily manufactured by micro machining method, and with higher reliability than the active measures. They found that such microchannel heat sinks could significantly improve the heat transfer performance. The microchannel heat sink with forward triangular offset ribs performs best as Reynolds numbers lower than 350, and the one with semicircular offset ribs yielded the best performance as Reynolds numbers higher than 400 . In order to obtain the insight into the fluid flow and heat transfer mechanisms and present a systematic and detailed analysis for such microchannel heat sink, the present paper focuses on the effects of geometry of both aligned and offset fan-shaped ribs on thermal and hydraulic characteristics. Three non-dimensional variables, representing the width, height and spacing 
of fan-shaped ribs are designed. As the first part of a three-part study, the objective of the paper is to identify the effects of such geometric and arrangement parameters on heat transfer, including temperature distribution, local and average heat transfer characteristics in such microchannel heat sink.

\section{Model descriptions}

\subsection{Computational domain and geometric configurations}

As illustrated in Fig. 1a, the computational domain has the length of $10 \mathrm{~mm}$, width of $0.25 \mathrm{~mm}$, and height of $0.35 \mathrm{~mm}$. The length and height of microchannel are $10 \mathrm{~mm}$ and 0.2 $\mathrm{mm}$, respectively. The width of the two parallel sidewalls for microchannel without ribs $\left(W_{\mathrm{c}}\right)$ is $0.1 \mathrm{~mm}$. The fan-shaped ribs are mounted on the two parallel sidewalls in tandem for both aligned and offset arrangements. The geometric parameters for fan-shaped ribs are shown in Fig. 1b, including the width $\left(W_{\mathrm{r}}\right)$, the height $\left(H_{\mathrm{r}}\right)$, and the spacing $\left(S_{\mathrm{r}}\right)$. The ranges for these geometric parameters examined in the present paper are 0.005-0.4 mm for $W_{\mathrm{r}}, 0.005-0.025$ $\mathrm{mm}$ for $H_{\mathrm{r}}$, and $0.2-5 \mathrm{~mm}$ for $S_{\mathrm{r}}$. These geometric parameters develop three non-dimensional variables, including the ratio of the width of rib to the spacing $\left(W_{\mathrm{r}} / S_{\mathrm{r}}\right)$, the ratio of the height of rib to the width of the two parallel sidewalls $\left(H_{\mathrm{r}} / W_{\mathrm{c}}\right)$, and the ratio of the spacing of ribs to the width of the two parallel sidewalls $\left(S_{\mathrm{r}} / W_{\mathrm{c}}\right)$. Meanwhile, a smooth microchannel heat sink without fan-shaped ribs is introduced for comparison.

\subsection{Governing equations and boundary conditions}

A three-dimensional solid-fluid conjugate model is used to predict heat transfer performance for such microchannel heat sink. The model employs the following assumptions: 
Developing flow and heat transfer in microchannels; Steady and laminar flow; Varied fluid thermal-physical properties; Viscous dissipation. The following governing equations are used in the mathematical modeling.

Continuity:

$$
\frac{\partial\left(\rho_{\mathrm{f}} u\right)}{\partial x}+\frac{\partial\left(\rho_{\mathrm{f}} v\right)}{\partial y}+\frac{\partial\left(\rho_{\mathrm{f}} w\right)}{\partial z}=0
$$

Momentum:

$$
\begin{aligned}
& \frac{\partial \rho_{\mathrm{f}} u u}{\partial x}+\frac{\partial \rho_{\mathrm{f}} u v}{\partial y}+\frac{\partial \rho_{\mathrm{f}} u w}{\partial z}=-\frac{\partial p}{\partial x}+\frac{\partial}{\partial x}\left(\mu_{\mathrm{f}} \frac{\partial u}{\partial x}\right)+\frac{\partial}{\partial y}\left(\mu_{\mathrm{f}} \frac{\partial u}{\partial y}\right)+\frac{\partial}{\partial z}\left(\mu_{\mathrm{f}} \frac{\partial u}{\partial z}\right) \\
& \frac{\partial \rho_{\mathrm{f}} v u}{\partial x}+\frac{\partial \rho_{\mathrm{f}} v v}{\partial y}+\frac{\partial \rho_{\mathrm{f}} v w}{\partial z}=-\frac{\partial p}{\partial y}+\frac{\partial}{\partial x}\left(\mu_{\mathrm{f}} \frac{\partial v}{\partial x}\right)+\frac{\partial}{\partial y}\left(\mu_{\mathrm{f}} \frac{\partial v}{\partial y}\right)+\frac{\partial}{\partial z}\left(\mu_{\mathrm{f}} \frac{\partial v}{\partial z}\right) \\
& \frac{\partial \rho_{\mathrm{f}} w u}{\partial x}+\frac{\partial \rho_{\mathrm{f}} w v}{\partial y}+\frac{\partial \rho_{\mathrm{f}} w w}{\partial z}=-\frac{\partial p}{\partial z}+\frac{\partial}{\partial x}\left(\mu_{\mathrm{f}} \frac{\partial w}{\partial x}\right)+\frac{\partial}{\partial y}\left(\mu_{\mathrm{f}} \frac{\partial w}{\partial y}\right)+\frac{\partial}{\partial z}\left(\mu_{\mathrm{f}} \frac{\partial w}{\partial z}\right)
\end{aligned}
$$

Energy equation for liquid:

$$
\frac{\partial \rho_{\mathrm{f}} u T}{\partial x}+\frac{\partial \rho_{\mathrm{f}} v T}{\partial y}+\frac{\partial \rho_{\mathrm{f}} w T}{\partial z}=\frac{\partial}{\partial x}\left(\frac{k_{\mathrm{f}}}{c_{\mathrm{pf}}} \frac{\partial T}{\partial x}\right)+\frac{\partial}{\partial y}\left(\frac{k_{\mathrm{f}}}{c_{\mathrm{pf}}} \frac{\partial T}{\partial y}\right)+\frac{\partial}{\partial z}\left(\frac{k_{\mathrm{f}}}{c_{\mathrm{pf}}} \frac{\partial T}{\partial z}\right)+\Phi
$$

In Eq. (5), $\Phi$ is the viscous dissipation term, which is written as

$$
\Phi=\mu_{\mathrm{f}}\left\{2\left[\left(\frac{\partial u}{\partial x}\right)^{2}+\left(\frac{\partial v}{\partial y}\right)^{2}+\left(\frac{\partial w}{\partial z}\right)^{2}\right]+\left(\frac{\partial u}{\partial y}+\frac{\partial v}{\partial x}\right)^{2}+\left(\frac{\partial u}{\partial z}+\frac{\partial w}{\partial x}\right)^{2}+\left(\frac{\partial v}{\partial z}+\frac{\partial w}{\partial y}\right)^{2}\right\}
$$

Energy equation for the solid

$$
\frac{\partial}{\partial x}\left(k_{\mathrm{s}} \frac{\partial T}{\partial x}\right)+\frac{\partial}{\partial y}\left(k_{\mathrm{s}} \frac{\partial T}{\partial y}\right)+\frac{\partial}{\partial z}\left(k_{\mathrm{s}} \frac{\partial T}{\partial z}\right)=0
$$

where $\rho$ is density, $\mu$ is dynamic viscosity, $c_{\mathrm{p}}$ is specific heat capacity, $k$ is thermal conductivity. Subscripts $f$ and s refer to fluid and solid, respectively.

For the sake of completeness, the boundary conditions corresponding to Fig.1a are mathematically listed as follows.

Velocity-inlet boundary: At the microchannel section, five inlet flow velocities $u_{\text {in }}$ are selected as the average velocity $\bar{u}$ in the constant cross-section region is respectively $1,2,3,4$ and 5 
$\mathrm{m} \cdot \mathrm{s}^{-1}$ and the corresponding Reynolds number is $187,316,443,582$ and 715 , respectively.

$x=0: u=u_{\text {in }}$ and $T_{\mathrm{f}}=T_{\text {in }}$

where $T_{\text {in }}=293 \mathrm{~K}$ is the given temperature at the channel inlet.

Pressure-outlet boundary: At the channel outlet, a constant pressure boundary condition is applied.

$x=10 \mathrm{~mm}: p_{\mathrm{f}}=p_{\text {out }}$

where $p_{\text {out }}=0$ (gauge pressure) is the given pressure at the channel outlet.

Constant heat flux boundary: At the base of computational domain, a constant heat flux of $q_{\mathrm{w}}$

$=10^{6} \mathrm{~W} \cdot \mathrm{m}^{-2}$ is imposed.

$z=0: \quad-k_{s} \frac{\partial T_{\mathrm{s}}}{\partial z}=q_{\mathrm{w}}$

Symmetry wall boundary: At the two sides of the computational domain, zero normal velocity and zero normal gradient of temperature for the symmetry planes.

$y=0, \frac{\partial T_{s}}{\partial y}=0$

$y=0.25 \mathrm{~mm}, \frac{\partial T_{\mathrm{s}}}{\partial y}=0$

Adiabatic boundary: For other surfaces, adiabatic boundary is applied.

$\frac{\partial T_{\mathrm{f}}}{\partial x}=0 \quad$ or $\quad \frac{\partial T_{\mathrm{s}}}{\partial x}=0$

In the present study, the water and silicon are used as fluid and solid for the computational domain, respectively. The properties of water are allowed to vary with temperature, and the functional relations between density, viscosity, specific heat and thermal conductivity with temperature are given as Incropera [16].

$$
\begin{aligned}
& \rho_{\mathrm{f}}=\frac{999.84+18.225 T-7.92 \times 10^{-3} T^{2}-5.545 \times 10^{-5} T^{3}+1.498 \times 10^{-7} T^{4}-3.933 \times 10^{-10} T^{5}}{1+1.816^{-2} T} \\
& \mu_{\mathrm{f}}=2.414 \times 10^{-5} \times 10^{\frac{247.8}{T-140}}
\end{aligned}
$$


$c_{\mathrm{pf}}=8958.9-40.535 T+0.11243 T^{2}-1.014 \times 10^{-4} T^{3}$

$k_{\mathrm{f}}=-0.58166+6.3556 \times 10^{-3} \mathrm{~T}-7.964 \times 10^{-6} \mathrm{~T}^{2}$

where the unit of $T$ is ${ }^{\circ} \mathrm{C}$ in Eq. (14) and $\mathrm{K}$ in Eqs. (15), (16) and (17). The thermal conductivity of silicon $k_{\mathrm{s}}$ is set as a constant of $148 \mathrm{~W} \cdot \mathrm{m}^{-1} \mathrm{~K}^{-1}$ in the computations.

\subsection{Solution methods and data acquisition}

In this paper, the numerical simulations are performed using the ANSYS FLUENT 12.0 software. The SIMPLEC algorithm is applied to solve the governing differential equations for the continuity, momentum and energy equations. The solutions are considered to be converged when the normalized residual values are less than $10^{-5}$ for all the variables. The numerical code is verified in a number of ways to ensure the validity. For every microchannel heat sink, a grid independence test is conducted using several different mesh sizes. The grid independence test is similar to Chai et al. [15]. Fig. 1c shows some grid generations in the $x-y$

plane $\left(z=0.25 \mathrm{~mm}, W_{\mathrm{c}}=0.1 \mathrm{~mm}, W_{\mathrm{r}}=0.1 \mathrm{~mm}, H_{\mathrm{r}}=0.025 \mathrm{~mm}\right.$ and $\left.S_{\mathrm{r}}=0.4 \mathrm{~mm}\right)$. In order to further check the accuracy and reliability of this mathematical model, numerical results of heat transfer for the smooth microchannel heat sink have been performed by comparing with the experimental results from Chai et al. [12]. As shown in Fig. 2, the comparisons are found to be in good agreement and the largest difference is no more than $8 \%$.

To present the numerical results of heat transfer characteristics, the important parameters are given below.

Reynolds number:

$$
\operatorname{Re}=\frac{\rho_{\mathrm{f}} \bar{u} D_{\mathrm{h}}}{\mu_{\mathrm{f}}}
$$


where $\rho_{\mathrm{f}}$ is the volume average fluid density, $\bar{u}$ is the average flow velocity as mentioned above, $D_{\mathrm{h}}$ is the hydraulic diameter calculated based on the constant cross-section region, $\mu_{\mathrm{f}}$ is the mass average fluid dynamic viscosity. The $\rho_{\mathrm{f}}$ and $\mu_{\mathrm{f}}$ are defined as

$$
\begin{aligned}
& \rho_{\mathrm{f}}=\frac{\int \rho_{\mathrm{f}, \mathrm{i}} d V}{V} \\
& \mu_{\mathrm{f}}=\frac{\int \mu_{\mathrm{f}, \mathrm{i}} \rho_{\mathrm{f}, \mathrm{i}} d V}{\int \rho_{\mathrm{f}, \mathrm{i}} d V}
\end{aligned}
$$

Local heat transfer coefficient and Nusselt number:

$$
\begin{aligned}
& h_{\mathrm{x}}=\frac{q_{\mathrm{w}} L W}{A\left(T_{\mathrm{w}, \mathrm{x}}-T_{\mathrm{f}, \mathrm{x}}\right)} \\
& N u_{\mathrm{x}}=\frac{h_{\mathrm{x}} D_{\mathrm{h}}}{k_{\mathrm{f}}}
\end{aligned}
$$

where $q_{\mathrm{w}}$ represents heat flux at the silicon base, $A$ is the contact surface area of water and silicon based on the smooth microchannel, $L$ and $W$ are respectively the length and width of computational domain, $k_{\mathrm{f}}$ is the mass average fluid thermal conductivity and defined as

$$
k_{\mathrm{f}}=\frac{\int k_{\mathrm{f}, \mathrm{i}} \rho_{\mathrm{f}, 1} d V}{\int \rho_{\mathrm{f}, \mathrm{i}} d V}
$$

$T_{\mathrm{w}, \mathrm{x}}$ and $T_{\mathrm{f}, \mathrm{x}}$ are the local heat sink base temperature and the local bulk fluid temperature, respectively, defined as

$$
\begin{aligned}
& T_{\mathrm{w}, \mathrm{X}}=\frac{\int T_{\mathrm{w}, \mathrm{x}, \mathrm{d}} d y}{\int d y}
\end{aligned}
$$

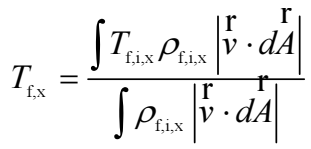

Average heat transfer coefficient and Nusselt number:

$$
\begin{aligned}
& \bar{h}=\frac{q_{\mathrm{w}} L W}{A\left(\overline{T_{\mathrm{w}}}-\overline{T_{\mathrm{f}}}\right)} \\
& \overline{N u}=\frac{\bar{h} D_{\mathrm{h}}}{k_{\mathrm{f}}}
\end{aligned}
$$


where $\overline{T_{\mathrm{w}}}$ and $\overline{T_{\mathrm{f}}}$ are the area-weighted temperature of the silicon base and the mass-average temperature of water in microchannels, respectively, defined as

$$
\begin{aligned}
& \overline{T_{\mathrm{w}}}=\frac{\int T_{\mathrm{w}, \mathrm{x}, \mathrm{y}} d y d x}{\int d y d x} \\
& \overline{T_{\mathrm{f}}}=\frac{\int T_{\mathrm{f}, \mathrm{x}, \mathrm{x}} \rho_{\mathrm{f}, \mathrm{i}, \mathrm{x}}|\mathrm{r} \cdot \stackrel{\mathrm{r}}{v} \cdot d A| d x}{\int \rho_{\mathrm{f}, \mathrm{x}}\left|{ }^{\mathrm{r}} \cdot d A\right| d x}
\end{aligned}
$$

Total thermal resistance:

$$
R_{\mathrm{th}}=\frac{\overline{T_{\mathrm{w}}}-T_{\mathrm{in}}}{q_{\mathrm{w}} L W}
$$

\section{Results and discussion}

\subsection{Parametric study on local heat transfer characteristics}

Flow structures should be firstly provided to present the physical issues behind the variation of heat transfer. Figure 3 shows the velocity contour and streamlines with $x$ in the range of 6.0 to $6.8 \mathrm{~mm}$ in the plane of $z=0.25 \mathrm{~mm}$ as $R e=443$. As it is bulky to depict the data for all the cases studied, for the purpose of comparison, results are presented here only for the microchannel heat sinks with fan-shaped ribs of $W_{\mathrm{r}}=0.1 \mathrm{~mm}, H_{\mathrm{r}}=0.025 \mathrm{~mm}$ and $S_{\mathrm{r}}=$ $0.4 \mathrm{~mm}$. For the smooth microchannel heat sink (MCHS), the region of maximum velocity is always at the channel center. After introduction of fan-shaped ribs to the channel, the region of maximum velocity is shifted. The flow entering and leaving the fan-shaped ribs region experience the flow passage constriction and expansion, which lead to the increase or decrease of water velocity due to the Bernoulli Effect. The water velocity obviously varies with the flow passage for the microchannel heat sink with aligned fan-shaped ribs (MCHS-AFR), and is not as obvious as MCHS-AFR for the microchannel heat sink with 
offset fan-shaped ribs (MCHS-OFR). From Fig. 3b, it is noted that the streamlines are interrupted by the aligned fan-shaped ribs, while some of them are interrupted and some change their direction under the influence of offset fan-shaped ribs. It is also found that at the region near the downstream surface of aligned fan-shaped ribs, the water velocity is very small and a recirculation is formed there. The interruption of streamlines results in heat transfer enhancement and higher pressure drop owing to the boundary layer redevelopment, while the recirculation leads to improvement or deterioration of heat transfer, which are dependent on the intensity of the second flow developed there. The interruption of streamlines depends on the flow passage ratio of constriction to expansion, while the recirculation size and the intensity of the second flow are dependent on both the geometry of fan-shaped ribs and the water hydraulic and thermo-physical properties. Therefore, the geometry of fan-shaped ribs and water velocity can have significant influence on thermal and hydraulic characteristics of such microchannel heat sink.

Figure 4 depicts the local temperature profiles with $x$ ranging from $6.0 \mathrm{~mm}$ to $6.8 \mathrm{~mm}$ in the plane of $z=0.25 \mathrm{~mm}$ as $R e=443$ with the same channel geometry as Fig. 3. It is noted that for the MCHS, the temperature in the channel center is lower, while shows higher values near the sidewalls, where there are large temperature gradients in the $y$ direction. After introduction of fan-shaped ribs to channel sidewalls, the temperature near the sidewalls obviously decreases, and the MCHS-OFR shows a smaller temperature difference between the channel center and the sidewalls than the MCHS-AFR. For MCHS-AFR, the isotherm varies mainly dependent on the periodically changed flow passage; For MCHS-OFR, the isotherm varies largely based on both the changed flow passage and the changed flow 
direction.

Figures $5 \mathrm{a}$ and $5 \mathrm{~b}$ respectively show the effects of $W_{\mathrm{r}}$ on $T_{\mathrm{w}, \mathrm{x}}$ and $N u_{\mathrm{x}}$ along the flow direction as $\operatorname{Re}=443$, where the non-dimensionalized length is defined as $\left(x^{*}=x /\left(D_{\mathrm{h}} \operatorname{RePr}\right)\right)$. The geometry of fan-shaped ribs used in these figures is $W_{\mathrm{c}}=0.1 \mathrm{~mm}, H_{\mathrm{r}}=0.025 \mathrm{~mm}, S_{\mathrm{r}}=$ $0.4 \mathrm{~mm}$ and $W_{\mathrm{r}}=0.1,0.2,0.3$ and $0.4 \mathrm{~mm}$. It is noticed that there are differences for $T_{\mathrm{w}, \mathrm{x}}$ and $N u_{\mathrm{x}}$ in the channel inlet, which is caused by the three-dimensional heat conduction in the silicon block. From Fig. 5a, it can be seen that the fan-shaped ribs can effectively prevent the increase of $T_{\mathrm{w}, \mathrm{x}}$ along the flow direction. Along the whole length of microchannel, the $T_{\mathrm{w}, \mathrm{x}}$ is increased by $20.8 \mathrm{~K}$ for MCHS, but just 9.5, 10.1, 10.2 and $10.6 \mathrm{~K}$ respectively for MCHS-AFR with $W_{\mathrm{r}} / S_{\mathrm{r}}=0.25,0.5,0.75$ and 1 , and $9.9,10.1,11.1$ and $11.5 \mathrm{~K}$ respectively for MCHS-OFR with $W_{\mathrm{r}} / S_{\mathrm{r}}=0.25,0.5,0.75$ and 1 . From Fig. $5 \mathrm{~b}$, it is noted that the fan-shaped ribs lead to obviously different local heat transfer characteristics. For the MCHS, the $N u_{\mathrm{x}}$ continually drops along the flow direction; For MCHS-AFR and MCHS-OFR, the drop of $N u_{\mathrm{x}}$ has been efficiently prevented and kept at a higher level. Further, as water flows across the aligned fan-shaped ribs, the $N u_{\mathrm{x}}$ rapidly increases or decreases with the contraction or expansion of flow passage, while there is no obvious fluctuation as water flows across the offset fan-shaped ribs. This phenomenon is mainly caused by the different ratio of contraction to expansion of flow passage, owing to the aligned or offset arrangement.

Figures $6 \mathrm{a}$ and $6 \mathrm{~b}$ show the effect of $H_{\mathrm{r}}$ on $T_{\mathrm{w}, \mathrm{x}}$ and $N u_{\mathrm{x}}$ along the flow direction as $R e=$ 443. The geometry parameter used in these figures are $W_{\mathrm{c}}=0.1 \mathrm{~mm}, S_{\mathrm{r}}=0.4 \mathrm{~mm}, W_{\mathrm{r}}=0.1$ $\mathrm{mm}$, and $H_{\mathrm{r}}=0.005,0.015$ and $0.025 \mathrm{~mm}$. From Fig. 6a, it can be seen that the $T_{\mathrm{w}, \mathrm{x}}$ at a given position largely decreases with the increase of $H_{\mathrm{r}} / W_{\mathrm{c}}$. Along the whole length of microchannel, 
the $T_{\mathrm{w}, \mathrm{x}}$ is increased by $17.4,12.1$ and $9.5 \mathrm{~K}$ respectively for MCHS-AFR with $H_{\mathrm{r}} / W_{\mathrm{c}}=0.05$, 0.15 and 0.25 , while by $17.6,12.5$ and $9.9 \mathrm{~K}$ respectively for MCHS-OFR with $H_{\mathrm{r}} / W_{\mathrm{c}}=0.05$, 0.15 and 0.25 . Correspondingly, the $N u_{\mathrm{x}}$ at a given position obviously increases with increase of $H_{\mathrm{r}} / W_{\mathrm{c}}$ as shown in Fig. 6b. Further, as $H_{\mathrm{r}} / W_{\mathrm{c}}=0.05$ and 0.15 , the $N u_{\mathrm{x}}$ continually drops along the flow direction and there is no obvious fluctuation as water flows across the fan-shaped ribs for both MCHS-AFR and MCHS-OFR, while as $H_{\mathrm{r}} / W_{\mathrm{c}}=0.25$, the $N u_{\mathrm{x}}$ for MCHS-AFR shows obvious fluctuation as water flows across the fan-shaped ribs. It is also found that there is larger $N u_{\mathrm{x}}$ difference between MCHS-AFR and MCHS-OFR at the middle region than the channel ends.

Because the fan-shaped ribs are used to provide shorter length required for repeated thermal developing flow, the spacing of ribs is another important geometry parameter to determine the local heat transfer characteristics. Figures $7 \mathrm{a}$ and $7 \mathrm{~b}$ show the effect of $S_{\mathrm{r}}$ on $T_{\mathrm{w}, \mathrm{x}}$ and $N u_{\mathrm{x}}$ along the flow direction as $R e=443$. The geometry parameters used in these figures are $W_{\mathrm{c}}=0.1 \mathrm{~mm}, W_{\mathrm{r}}=0.1 \mathrm{~mm}, H_{\mathrm{r}}=0.025 \mathrm{~mm}$ and $S_{\mathrm{r}}=2,1,0.5$, and $0.25 \mathrm{~mm}$. From Fig. $7 \mathrm{a}$, it can be found that the $T_{\mathrm{w}, \mathrm{x}}$ at a given position largely decreases with the increase of $S_{\mathrm{r}} / W_{\mathrm{c}}$. Along the whole length of microchannel, the $T_{\mathrm{w}, \mathrm{x}}$ is increased by $15.1,11.7,10$ and 9.3 $\mathrm{K}$ respectively for MCHS-AFR with $S_{\mathrm{r}} / W_{\mathrm{c}}=20,10,5$ and 2.5, and 16.2, 13.5, 11.4 and $9.8 \mathrm{~K}$ respectively for MCHS-OFR with $S_{\mathrm{r}} / W_{\mathrm{c}}=20,10,5$ and 2.5. Correspondingly, the $N u_{\mathrm{x}}$ at a given position obviously increases with decrease of $S_{\mathrm{r}} / W_{\mathrm{c}}$ as shown in Fig. 7b. As $S_{\mathrm{r}} / W_{\mathrm{c}}=20$ and 10 , the $N u_{\mathrm{x}}$ continually drops along the flow direction; As $S_{\mathrm{r}} / W_{\mathrm{c}}=5$, the $N u_{\mathrm{x}}$ just drops near the channel inlet and then keeps at a higher level; As $S_{\mathrm{r}} / W_{\mathrm{c}}=2.5$, the $N u_{\mathrm{x}}$ keeps at a higher level without obvious drop from the channel inlet. It is also noted that for $S_{\mathrm{r}} / W_{\mathrm{c}}=20$ 
and 10, the MCHS-AFR shows a little higher $N u_{\mathrm{x}}$ at a given position than MCHS-OFR; For $S_{\mathrm{r}} / W_{\mathrm{c}}=5$, the MCHS-AFR shows a little lower $N u_{\mathrm{x}}$ than MCHS-OFR as $x^{*}<0.005$, while a contrary tendency as $x^{*}>0.005$; For $S_{\mathrm{r}} / W_{\mathrm{c}}=2.5$, the MCHS-AFR and MCHS-OFR show almost the same $N u_{\mathrm{x}}$ level as $x^{*}>0.01$, and as $x^{*}<0.01$, the MCHS-OFR performs a little better.

\subsection{Parametric study on average heat transfer characteristics}

Figure 8 shows the effect of $R e$ and $W_{\mathrm{r}} / S_{\mathrm{r}}$ on $\overline{N u}, \overline{N u} / \overline{N u_{0}}$ and $R_{\mathrm{th}} / R_{\mathrm{th}, 0}$, where $\overline{N u_{0}}$ and $R_{\mathrm{th}, 0}$ are the average Nusselt number and total thermal resistance of the baseline MCHS. The geometry of fan-shaped ribs used in these figures is $W_{\mathrm{c}}=0.1 \mathrm{~mm}, H_{\mathrm{r}}=0.025 \mathrm{~mm}, S_{\mathrm{r}}=0.4$ $\mathrm{mm}$ and $W_{\mathrm{r}}=0.05-0.4 \mathrm{~mm}$. It can be noted that the offset ribs can give rise to significant heattransfer enhancement. From Fig. 8a, it can be seen that the $\overline{N u}$ obviously increases with increase of $R e$. With the increase of $R e$ from 187 to 715 , the $\overline{N u}$ increases from 4.96 to 6.91 for MCHS; from 7.59 to $12.82,8.32$ to $12.42,8.66$ to 12.29 and 9.34 to 11.52 respectively for MCHS-AFR with $W_{\mathrm{r}} / S_{\mathrm{r}}=0.25,0.5,0.75$ and 1 ; from 7.58 to $12.86,7.52$ to $12.12,7.95$ to 11.58 and 8.55 to 11.13 respectively for MCHS-OFR with $W_{\mathrm{r}} / S_{\mathrm{r}}=0.25,0.5,0.75$ and 1 . From Fig. $8 \mathrm{~b}$, it can be seen that there is no very clear variation tendency of $\overline{N u} / \overline{N u}_{0}$ with $W_{\mathrm{r}} / S_{\mathrm{r}}$. With the increase of $W_{\mathrm{r}} / S_{\mathrm{r}}$ form 0.125 to 1 , the $\overline{N u} / \overline{N u}_{0}$ for MCHS-AFR changes in the ranges of 1.49-1.88, 1.64-1.89, 1.73-1.89, 1.73-1.86 and 1.67-1.86 respectively for $R e=187,316$, 443, 582 and 715, and the $\overline{N u} / \overline{N u}_{0}$ for MCHS-OFR changes in the ranges of 1.51-1.72, $1.62-1.88,1.63-1.95,1.61-1.91$ and 1.61-1.89 respectively for $R e=187,316,443,582$ and 715. From Fig. 8c, it can be found that the increase of $R e$ results in obviously lower $R_{\mathrm{th}} / R_{\mathrm{th}, 0}$, 
but the $R_{\mathrm{th}} / R_{\mathrm{th}, 0}$ does not change much with $W_{\mathrm{r}} / S_{\mathrm{r}}$. As $R e=187$ and 316, with the increase of $W_{\mathrm{r}} / S_{\mathrm{r}}$, the $R_{\mathrm{th}} / R_{\mathrm{th}, 0}$ generally increases and then drops very slowly. As $R e=443,582$ and 715, the difference of $R_{\mathrm{th}} / R_{\mathrm{th}, 0}$ is smaller with changed $W_{\mathrm{r}} / S_{\mathrm{r}}$. With the increase of $W_{\mathrm{r}} / S_{\mathrm{r}}$ from 0.125 to 1 , the $R_{\mathrm{th}} / R_{\mathrm{th}, 0}$ changes in the ranges of $0.72-0.8,0.66-0.72,0.62-0.67,0.62-0.66$ and 0.61-0.66 respectively for MCHS-AFR as $R e=187,316,443,582$ and 715, and 0.75-0.8, $0.66-0.72,0.62-0.69,0.6-0.68$ and $0.6-0.68$ respectively for MCHS-AFR as $R e=187,316$, 443,582 and 715.

Figure 9 shows the effect of $R e$ and $H_{\mathrm{r}} / W_{\mathrm{c}}$ on $\overline{N u}, \overline{N u} / \overline{N u}_{0}$ and $R_{\mathrm{th}} / R_{\mathrm{th}, 0}$. The geometry of fan-shaped ribs used in these figures is $W_{\mathrm{c}}=0.1 \mathrm{~mm}, S_{\mathrm{r}}=0.4 \mathrm{~mm}, W_{\mathrm{r}}=0.1 \mathrm{~mm}$ and $H_{\mathrm{r}}=$ 0.005-0.025 mm. From Fig. 9a, it can be seen that the increase of $H_{\mathrm{r}} / W_{\mathrm{c}}$ leads to large increase of $\overline{N u}$. As $H_{\mathrm{r}} / W_{\mathrm{c}}=0.05$ and 0.15 , the MCHS-AFR shows a little higher $\overline{N u}$ than MCHS-OFR, while as $H_{\mathrm{r}} / W_{\mathrm{c}}=0.25$, they are almost the same. With the increase of $R e$ from 187 to 715 , the $\overline{\mathrm{Nu}}$ increases from 5.5 to $7.8,6.07$ to 11.89 and 7.59 to 12.82 respectively for MCHS-AFR with $H_{\mathrm{r}} / W_{\mathrm{c}}=0.05,0.15$ and 0.25 ; from 5.39 to $7.38,5.88$ to 10.69 and 7.58 to 12.86 respectively for MCHS-OFR with $H_{\mathrm{r}} / W_{\mathrm{c}}=0.05,0.15$ and 0.25 . From Fig. $9 \mathrm{~b}$, it is noticed that for the increase of $H_{\mathrm{r}} / W_{\mathrm{c}}$ generally leads to increase of $\overline{N u} / \overline{N u}_{0}$. Comparing of $\overline{N u} / \overline{N u}_{0}$ indicates that the MCHS-AFR results in better heat transfer characteristics at lower $H_{\mathrm{r}} / W_{\mathrm{c}}$. With the increase of $H_{\mathrm{r}} / W_{\mathrm{c}}$ from 0.05 to 0.25 , the $\overline{N u} / \overline{N u_{0}}$ increases from 1.11 to 1.53 , 1.13 to $1.7,1.13$ to $1.82,1.13$ to 1.82 and 1.13 to 1.86 for MCHS-AFR respectively as $R e=$ $187,316,443,582$ and 715 ; from 1.09 to $1.53,1.09$ to $1.69,1.08$ to $1.78,1.07$ to 1.86 for MCHS-OFR respectively as $R e=187,316,443,582$ and 715. From Fig. 9c, it can be found that the increases of $R e$ and $H_{\mathrm{r}} / W_{\mathrm{c}}$ lead to lower $R_{\mathrm{th}} / R_{\mathrm{th}, 0}$. MCHS-AFR shows lower $R_{\mathrm{th}} / R_{\mathrm{th}, 0}$ 
than MCHS-OFR as $H_{\mathrm{r}} / W_{\mathrm{c}}<0.25$, while almost the same as $H_{\mathrm{r}} / W_{\mathrm{c}}=0.25$. With the increase of $H_{\mathrm{r}} / W_{\mathrm{c}}$ from 0.05 to 0.25 , the $R_{\mathrm{th}} / R_{\mathrm{th}, 0}$ decreases from 0.94 to $0.79,0.92$ to $0.7,0.9$ to $0.65,0.9$ to 0.63 and 0.9 to 0.61 for MCHS-AFR respectively as $R e=187,316,443,582$ and 715 ; from 0.95 to $0.79,0.94$ to $0.7,0.94$ to $0.66,0.94$ to 0.63 and 0.95 to 0.61 for MCHS-OFR respectively as $R e=187,316,443,582$ and 715 .

Figure 10 shows the effect of $R e$ and $S_{\mathrm{r}} / W_{\mathrm{c}}$ on $\overline{N u}, \overline{N u} / \overline{N u}_{0}$ and $R_{\mathrm{th}} / R_{\mathrm{th}, 0}$. The geometry of fan-shaped ribs used in these figures is $W_{\mathrm{c}}=0.1 \mathrm{~mm}, W_{\mathrm{r}}=0.1 \mathrm{~mm}, H_{\mathrm{r}}=0.025 \mathrm{~mm}$ and $S_{\mathrm{r}}=$ 0.2-5 mm. The number of fan-shaped ribs and the corresponding value of $S_{\mathrm{r}} / W_{\mathrm{c}}$ are listed in Table 1. From Fig. 10a, it is noted that the decrease of $S_{\mathrm{r}} / W_{\mathrm{c}}$ leads to large increase of $\overline{N u}$. As $S_{\mathrm{r}} / W_{\mathrm{c}}=20$ and 10 , the MCHS-AFR shows a little higher $\overline{N u}$ than MCHS-OFR, while as $S_{\mathrm{r}} / W_{\mathrm{c}}$ $=5$ and 2.5, the MCHS-OFR shows larger $\overline{N u}$ than MCHS-AFR as $R e<443$ and the contrary tendency as $R e>443$. With the increase of $R e$ from 187 to 715 , the $\overline{N u}$ increases from 5.62 to 9.56, 6.28 to $10.98,7.22$ to 12.09 and 8.32 to 13.31 respectively for MCHS-AFR with $S_{\mathrm{r}} / W_{\mathrm{c}}=$ $20,10,5$ and $2.5 ; 5.45$ to $8.49,5.92$ to $10.2,7.19$ to 11.68 and 8.71 to 12.86 respectively for MCHS-AFR with $S_{\mathrm{r}} / W_{\mathrm{c}}=20,10,5$ and 2.5. From Fig. $10 \mathrm{~b}$, it can be found that the $\overline{N u} / \overline{N u_{0}}$ generally increases with the decrease of $S_{\mathrm{r}} / W_{\mathrm{c}}$, except the cases for MCHS-AFR with $S_{\mathrm{r}} / W_{\mathrm{c}}=2$ at $R e=582$ and 715. As $S_{\mathrm{r}} / W_{\mathrm{c}}>5$, the MCHS-AFR shows higher $\overline{N u} / \overline{N u_{0}}$ than MCHS-OFR, while there is no obvious tendency as $S_{\mathrm{r}} / W_{\mathrm{c}}<5$. With the decrease of $S_{\mathrm{r}} / W_{\mathrm{c}}$ form 50 to 2 , the $\overline{N u} / \overline{N u}_{0}$ increases from 1.05 to $1.89,1.08$ to $2.01,1.1$ to $1.98,1.12$ to 1.91 and 1.16 to 1.91 for MCHS-AFR respectively as $R e=187,316,443,582$ and 715 ; from 1.04 to $1.81,1.06$ to $2.01,1.08$ to $2.03,1.11$ to 1.99 and 1.13 to 1.97 for MCHS-OFR respectively as $R e=187,316,443,582$ and 715 . Correspondingly the increases of $R e$ and decrease of 
$S_{\mathrm{r}} / W_{\mathrm{c}}$ lead to lower $R_{\mathrm{th}} / R_{\mathrm{th}, 0}$ as shown in Fig. $10 \mathrm{c}$. With the decrease of $S_{\mathrm{r}} / W_{\mathrm{c}}$ from 50 to 2 , the $R_{\mathrm{th}} / R_{\mathrm{th}, 0}$ decreases from 0.96 to $0.71,0.94$ to $0.64,0.93$ to $0.61,0.91$ to 0.61 and 0.89 to 0.6 for MCHS-AFR respectively as $R e=187,316,443,582$ and 715 ; from 0.98 to $0.73,0.96$ to 0.63 , 0.94 to $0.6,0.92$ to 0.6 and 0.91 to 0.58 for MCHS-OFR respectively as $R e=187,316,443$, 582 and 715.

Based on the numerical results, the correlations of heat transfer for the microchannel heat sinks with different fan-shaped ribs on sidewalls are deduced by least squares method and shown as follows:

MCHS-AFR: $\overline{N u}=1.898 \operatorname{Re}^{0.2939} \operatorname{Pr}^{0.3}\left(W_{\mathrm{r}} / S_{\mathrm{r}}\right)^{-0.0027}\left(H_{\mathrm{r}} / W_{\mathrm{c}}\right)^{0.2404}\left(S_{\mathrm{r}} / W_{\mathrm{c}}\right)^{-0.1684}$

MCHS-OFR: $\overline{N u}=2.4145 \operatorname{Re}^{0.2782} \operatorname{Pr}^{0.3}\left(W_{\mathrm{r}} / S_{\mathrm{r}}\right)^{-0.0285}\left(H_{\mathrm{r}} / W_{\mathrm{c}}\right)^{0.3304}\left(S_{\mathrm{r}} / W_{\mathrm{c}}\right)^{-0.2428}$

for $187 \leq R e \leq 715, \quad 4.87 \leq \operatorname{Pr} \leq 6.18, \quad 0.02 \leq W_{\mathrm{r}} / S_{\mathrm{r}} \leq 1, \quad 0.05 \leq H_{\mathrm{r}} / W_{\mathrm{c}} \leq 0.25$, $2 \leq S_{\mathrm{r}} / W_{\mathrm{c}} \leq 50$.

Figure 11 compares predictions of these correlations with the present CFD (Computational Fluid Dynamics) simulated data. The predictive accuracy of the correlations is measured by mean absolute error, which is defined as

MAE $=\frac{1}{N} \sum_{i=1}^{N} \frac{\left|\overline{N u}_{\text {pred }}-\overline{N u}_{\text {cfd }}\right|}{\overline{N u}_{\text {cfd }}} \times 100 \%$

It can be found excellent agreement between simulated and predicted values, with $\mathrm{MAE}=$ $2.5 \%$ for MCHS-AFR and MAE $=3.8 \%$ for MCHS-OFR.

\section{Conclusions}

Three-dimensional numerical simulations have been conducted for laminar flow and heat transfer in the microchannel heat sinks with different fan-shaped ribs on sidewalls. The effect 
of different geometric parameters such as the width, height and spacing of fan-shaped ribs, aligned or offset arrangements are comprehensively studied. Based on the simulated results, the conclusions can be summarized as follows:

(1). The fan-shaped ribs can effectively prevent the increase of $T_{\mathrm{w}, \mathrm{x}}$ along the flow direction and keep $N u_{\mathrm{x}}$ at a higher level. The $N u_{\mathrm{x}}$ for MCHS-AFR more easily shows obvious fluctuation as water flows across the fan-shaped ribs due to the largely changed flow passage. The height and spacing of fan-shaped ribs have significant influences on the heat transfer characteristics, while the width of ribs shows less. The increase of height and decrease of spacing of fan-shaped ribs can significantly improve the local and average heat transfer coefficients.

(2). For the microchannel heat sinks with lower height and larger spacing of fan-shaped ribs, the aligned arrangement shows higher heat transfer coefficient than the offset one. For Reynolds number ranging from 187 to 715 and the studied geometric parameters of fan-shaped ribs, there are a 6-101\% increase in average Nusselt number and a 3-40\% decrease in total thermal resistance for MCHS-AFR, while a $4-103 \%$ increase in average Nusselt number and a $2-42 \%$ decrease in total thermal resistance for MCHS-OFR, comparing with the smooth one. Thus, such microchannel heat sink can be proposed as next generation cooling devices for removing high heat flux instead of using conventional smooth microchannel heat sink.

(3). New heat transfer correlations for the microchannel heat sinks with different fan-shaped ribs on sidewalls are proposed, which fit the present CFD simulated data with a mean absolute error of 2.5\% for MCHS-AFR 3.8\% for MCHS-OFR.. 


\section{Acknowledgements}

The work was supported by the Engineering and Physical Sciences Research Council (EPSRC) of the UK through research grant (EP/L001233/1) and the National Natural Science

Foundation of China (51576005). 


\section{References}

[1] D.B. Tuckerman, R.F.W. Pease, High-performance heat sinking for VLSI, Electronic Device Letters 2 (1981) 126-129.

[2] J.L. Xu, Y.H. Gan, D.C. Zhang, Microscale heat transfer enhancement using thermal boundary layer redeveloping concept, International Journal of Heat and Mass Transfer $48(2005) 1662-1674$.

[3] J.L. Xu, Y.X. Song, W Zhang, Numerical simulations of interrupted and conventional microchannel heat sinks, International Journal of Heat and Mass Transfer 51 (2008) $5906-5917$.

[4] L. Chai, G.D. Xia, M.Z. Zhou, Optimum thermal design of interrupted microchannel heat sink with rectangular ribs in the transverse microchambers, Applied Thermal Engineering 51 (2013) 880-989.

[5] Y.J. Cheng, Numerical simulation of stacked microchannel heat sink with mixing-enhanced passive structure, International Communications in Heat and Mass Transfer 34 (2007) 295-303.

[6] P. Promvonge, S. Sripattanapipat, S. Kwankaomeng, Laminar periodic flow and heat transfer in square channel with $45^{\circ}$ inline baffles on two opposite walls, International Journal of Thermal Sciences 49 (2010) 963-975.

[7] C. Liu, J. Teng, J.C. Chu, Experimental investigations on liquid flow and heat transfer in rectangular microchannel with longitudinal vortex generators, International Journal 
of Heat and Mass Transfer 54 (2011) 3069-3080.

[8] A. Ebrahimi, R. Ehsan Roohi, K. Saeid, Numerical study of liquid flow and heat transfer in rectangular microchannel with longitudinal vortex generators, Applied Thermal Engineering 78 (2015) 576-583.

[9] L. Chai, G.D. Xia, M.Z. Zhou, Numerical simulation of fluid flow and heat transfer in a microchannel heat sink with offset fan-shaped reentrant cavities in sidewall, International Communications in Heat and Mass Transfer 38 (2011) 577-584.

[10] G.D. Xia, L. Chai, M.Z. Zhou, Effects of structural parameters on fluid flow and heat transfer in a microchannel with aligned fan-shaped reentrant cavities, International Journal of Thermal Sciences 50 (2011) 411-419.

[11] G.D. Xia, L. Chai, H.Y. Wang, Optimum thermal design of microchannel heat sink with triangular reentrant cavities, Applied Thermal Engineering 31 (2011) 1208-1219.

[12] L. Chai, G.D. Xia, L. Wang, M.Z. Zhou, Z.Z. Cui, Heat transfer enhancement in microchannel heat sinks with periodic expansion-constriction cross-sections, International Journal of Heat and Mass Transfer 62 (2013) 741-751.

[13] A.J. Foong, N. Ramesh, T.T. Chandratilleke, Laminar convective heat transfer in a microchannel with internal longitudinal fins, International Journal of Thermal Sciences 48 (2009) 1908-1913.

[14] G. Xie, H Shen, C.C. Wang, Parametric study on thermal performance of microchannel heat sinks with internal vertical Y-shaped bifurcations, International Journal of Heat and Mass Transfer 90 (2015) 948-958.

[15] L. Chai, G.D. Xia, H.S. Wang, Numerical study of laminar flow and heat transfer in 
microchannel heat sink with offset ribs on sidewalls, Applied Thermal Engineering 92 (2016) 32-41.

[16] F. Incropera, Liquid Cooling of Electronic Devices by Single-Phase Convection, Wiley, 1999. 


\section{Nomenclature}

A

Area, $\mathrm{m}^{2}$

$c_{p}$

Specific heat capacity, $\mathrm{J} \cdot \mathrm{kg}^{-1} \mathrm{~K}^{-1}$

$D_{\mathrm{h}}$

Hydraulic diameter, $m$

$h$

Heat transfer coefficient, $\mathrm{W} \cdot \mathrm{m}^{-2} \cdot \mathrm{K}^{-1}$

$\bar{h}$

Average heat transfer coefficient, $\mathrm{W} \cdot \mathrm{m}^{-2} \cdot \mathrm{K}^{-1}$

$H$

Height, $m$

$k$

Thermal conductivity, $\mathrm{W} \cdot \mathrm{m}^{-1} \cdot \mathrm{K}^{-1}$

$L$

Length, $m$

MAE

mean absolute error

$N u$

Nusselt number

$\overline{N u}$

Average Nusselt number

$\overline{N u}_{0}$

Average Nusselt number for the smooth microchannel heat sink

$p$

Pressure, $\mathrm{Pa}$

Pr

Prandtl number

$q$

Heat flux, $\mathrm{W} \cdot \mathrm{m}^{-2}$

$R_{\text {th }}$

Total thermal resistance, $\mathrm{K} \cdot \mathrm{W}^{-1}$

$R_{\mathrm{th}, 0}$

Total thermal resistance for the smooth microchannel heat sink, $\mathrm{K} \cdot \mathrm{W}^{-1}$

Re

Reynolds number

$T$

Temperature, K

$\bar{T}$

Average temperature, $\mathrm{K}$ 


\begin{tabular}{|c|c|}
\hline$u, v, w$ & Velocity components in $x, y$ and $z$ directions, $\mathrm{m} \cdot \mathrm{s}^{-1}$ \\
\hline $\bar{u}$ & Average velocity, $\mathrm{m} \cdot \mathrm{s}^{-1}$ \\
\hline$V$ & Volume, $\mathrm{m}^{3}$ \\
\hline$W$ & Width, m \\
\hline$x, y, z$ & Cartesian coordinates, $\mathrm{m}$ \\
\hline \multicolumn{2}{|c|}{ Greek letters } \\
\hline$\rho$ & Density, $\mathrm{kg} \cdot \mathrm{m}^{-3}$ \\
\hline$\mu$ & Dynamic viscosity, $\mathrm{Pa} \cdot \mathrm{s}$ \\
\hline$\Phi$ & Viscous dissipation term \\
\hline \multicolumn{2}{|c|}{ Subscripts } \\
\hline $\mathrm{c}$ & Channel \\
\hline $\mathrm{f}$ & Fluid \\
\hline in & Inlet \\
\hline out & Outlet \\
\hline $\mathrm{r}$ & Rib \\
\hline s & Solid \\
\hline $\mathrm{w}$ & Silicon base \\
\hline
\end{tabular}


Table. 1 The number of fan-shaped ribs on each sidewall and the corresponding value of $S_{\mathrm{r}}$.

\begin{tabular}{cccccccccccc}
\hline Number & 2 & 4 & 5 & 8 & 10 & 16 & 20 & 25 & 32 & 40 & 50 \\
\hline$S_{\mathrm{r}} / W_{\mathrm{c}}$ & 50 & 25 & 20 & 12.5 & 10 & 6.25 & 5 & 4 & 3.125 & 2.5 & 2 \\
\hline
\end{tabular}




\section{Figure captions}

Fig. 1 Microchannel heat sinks with fan-shaped ribs on sidewalls. (a) Schematic diagram of the computational domain. (b) Geometric parameters and arrangement of fan-shaped ribs. (c) Grid generations in the $x-y$ plane $\left(z=0.25 \mathrm{~mm}, W_{\mathrm{c}}=0.1 \mathrm{~mm}, W_{\mathrm{r}}=0.1 \mathrm{~mm}, H_{\mathrm{r}}=0.025 \mathrm{~mm}\right.$ and $\left.S_{\mathrm{r}}=0.4 \mathrm{~mm}\right)$.

Fig. 2 Verification of mathematical model for Nusselt number in smooth microchannel heat sink.

Fig. 3 Velocity contour and streamlines $\left(R e=443, z=0.25 \mathrm{~mm}, W_{\mathrm{c}}=0.1 \mathrm{~mm}, W_{\mathrm{r}}=0.1 \mathrm{~mm}\right.$, $H_{\mathrm{r}}=0.025 \mathrm{~mm}$ and $S_{\mathrm{r}}=0.4 \mathrm{~mm}$ ). (a) Velocity contour. (b) streamlines.

Fig. 4 Temperature distribution $\left(R e=443, z=0.25 \mathrm{~mm}, W_{\mathrm{c}}=0.1 \mathrm{~mm}, W_{\mathrm{r}}=0.1 \mathrm{~mm}, H_{\mathrm{r}}=\right.$ $0.025 \mathrm{~mm}$ and $\left.S_{\mathrm{r}}=0.4 \mathrm{~mm}\right)$.

Fig. 5 Effects of fan-shaped rib's width on local heat transfer characteristics $\left(R e=443, W_{\mathrm{c}}=\right.$ $0.1 \mathrm{~mm}, H_{\mathrm{r}}=0.025 \mathrm{~mm}$ and $S_{\mathrm{r}}=0.4 \mathrm{~mm}$ ). (a) $T_{\mathrm{w}, \mathrm{x}}$ versus $x$. (b) $N u_{\mathrm{x}}$ versus $x^{*}$.

Fig. 6 Effects of fan-shaped rib's height on local heat transfer characteristics $\left(R e=443, W_{\mathrm{c}}\right.$ $=0.1 \mathrm{~mm}, S_{\mathrm{r}}=0.4 \mathrm{~mm}$ and $W_{\mathrm{r}}=0.1 \mathrm{~mm}$ ). (a) $T_{\mathrm{w}, \mathrm{x}}$ versus $x$. (b) $N u_{\mathrm{x}}$ versus $x^{*}$.

Fig. 7 Effects of fan-shaped rib's spacing on local heat transfer characteristics $\left(R e=443, W_{\mathrm{c}}\right.$ $=0.1 \mathrm{~mm}, W_{\mathrm{r}}=0.1 \mathrm{~mm}$ and $H_{\mathrm{r}}=0.025 \mathrm{~mm}$ ). (a) $T_{\mathrm{w}, \mathrm{x}}$ versus $x$. (b) $N u_{\mathrm{x}}$ versus $x^{*}$.

Fig. 8 Effects of fan-shaped rib's width on average heat transfer characteristics $\left(W_{\mathrm{c}}=0.1\right.$ $\mathrm{mm}, H_{\mathrm{r}}=0.025 \mathrm{~mm}$ and $S_{\mathrm{r}}=0.4 \mathrm{~mm}$ ). (a) $\overline{N u}$ versus $R e$. (b) $\overline{N u} / \overline{N u}_{0}$ versus $W_{\mathrm{r}} / S_{\mathrm{r}}$. (c) $R_{\mathrm{th}} / R_{\mathrm{th}, 0}$ versus $W_{\mathrm{r}} / S_{\mathrm{r}}$.

Fig. 9 Effects of fan-shaped rib's height on average heat transfer characteristics $\left(W_{\mathrm{c}}=0.1\right.$ $\mathrm{mm}, S_{\mathrm{r}}=0.4 \mathrm{~mm}$ and $W_{\mathrm{r}}=0.1 \mathrm{~mm}$ ). (a) $\overline{N u}$ versus $R e$. (b) $\overline{N u} / \overline{N u}_{0}$ versus $H_{\mathrm{r}} / W_{\mathrm{c}}$. (c) $R_{\mathrm{th}} / R_{\mathrm{th}, 0}$ 
versus $H_{\mathrm{r}} / W_{\mathrm{c}}$.

Fig. 10 Effects of fan-shaped rib's spacing on average heat transfer characteristics $\left(W_{\mathrm{c}}=0.1\right.$ mm, $W_{\mathrm{r}}=0.1 \mathrm{~mm}$ and $H_{\mathrm{r}}=0.025 \mathrm{~mm}$ ). (a) $\overline{N u}$ versus $R e$. (b) $\overline{N u} / \overline{N u}_{0}$ versus $S_{\mathrm{r}} / W_{\mathrm{c}}$. (c) $R_{\mathrm{th}} / R_{\mathrm{th}, 0}$ versus $S_{\mathrm{r}} / W_{\mathrm{c}}$.

Fig. 11 Comparison of predictions of heat transfer correlations with the present CFD simulated data. (a) MCHS-AFR. (b) MCHS-OFR. 
Fig. 1a and $1 \mathrm{~b}$

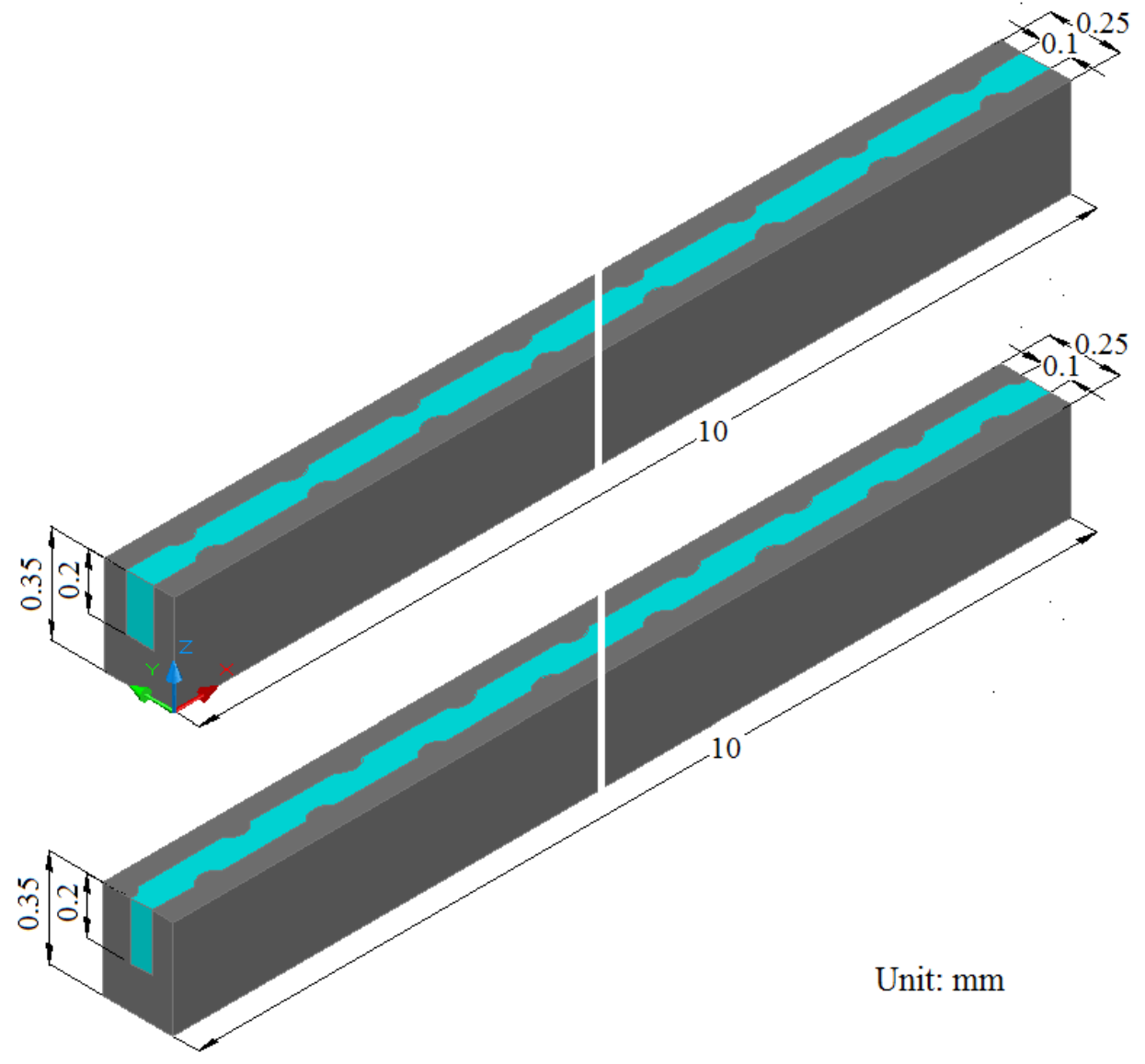

(a)
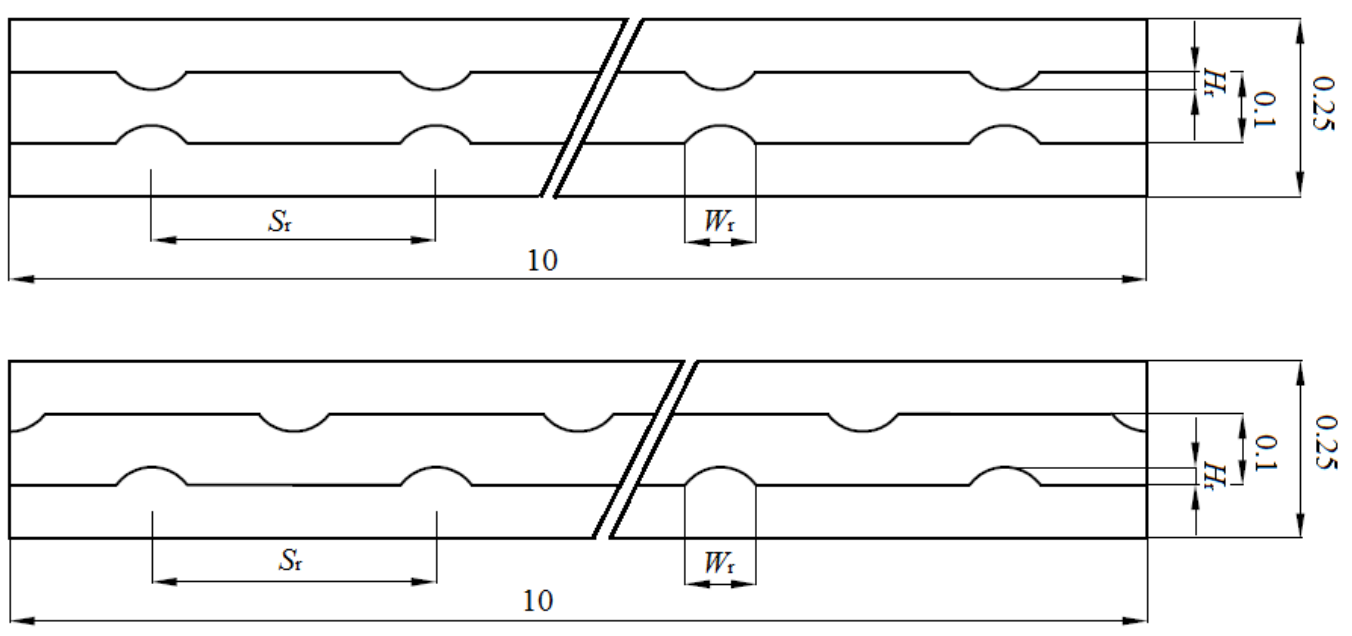

Unit: $\mathrm{mm}$

(b) 
Fig. 1c

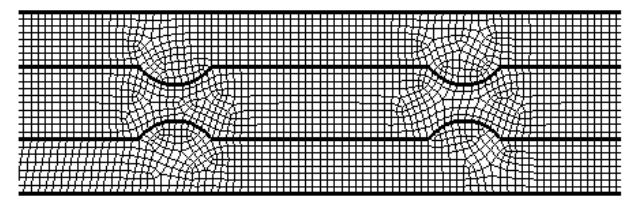

MCHS-AFR

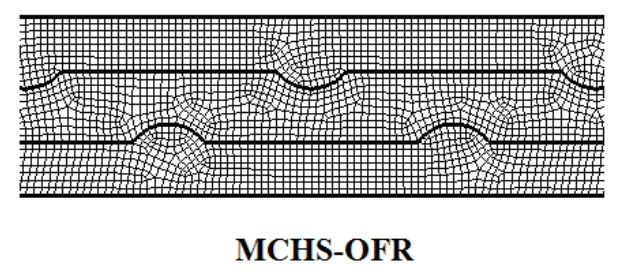

(c) 
Fig. 2

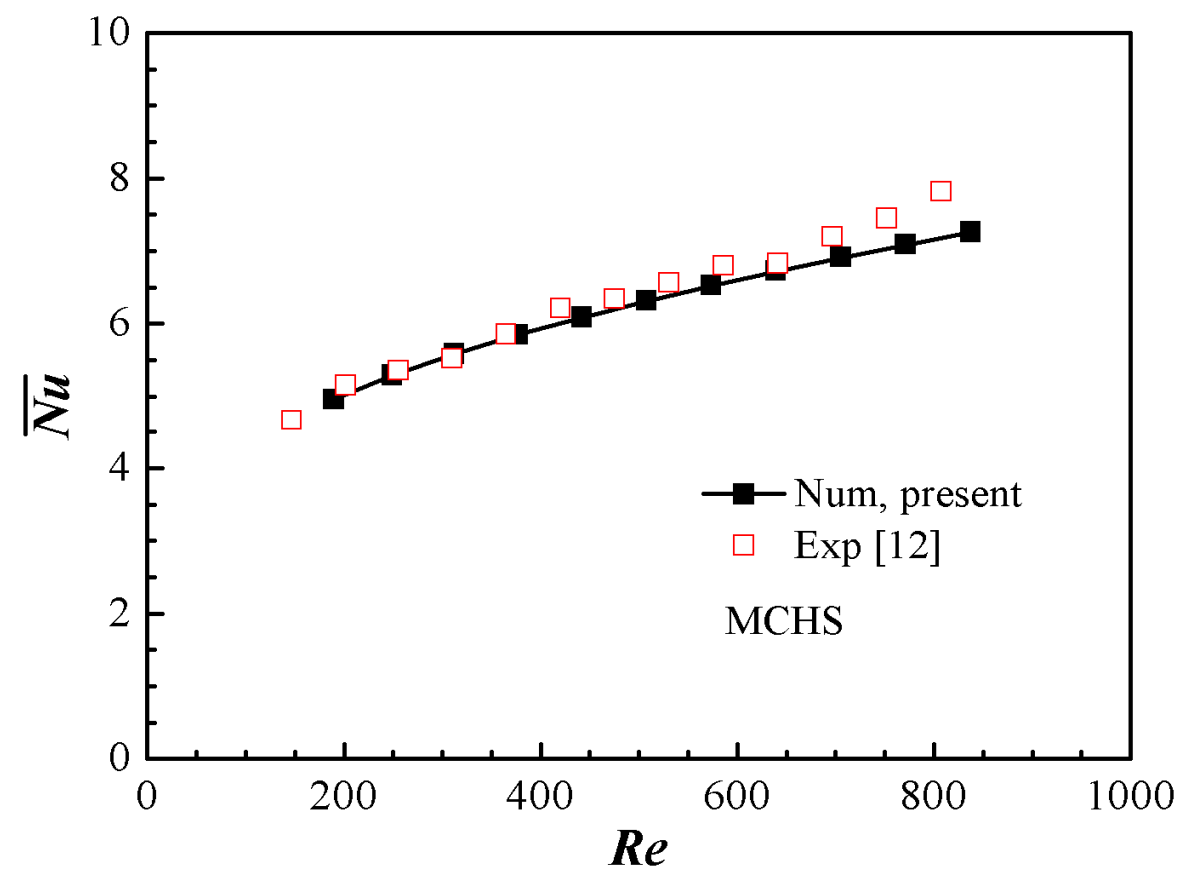


Fig. 3a

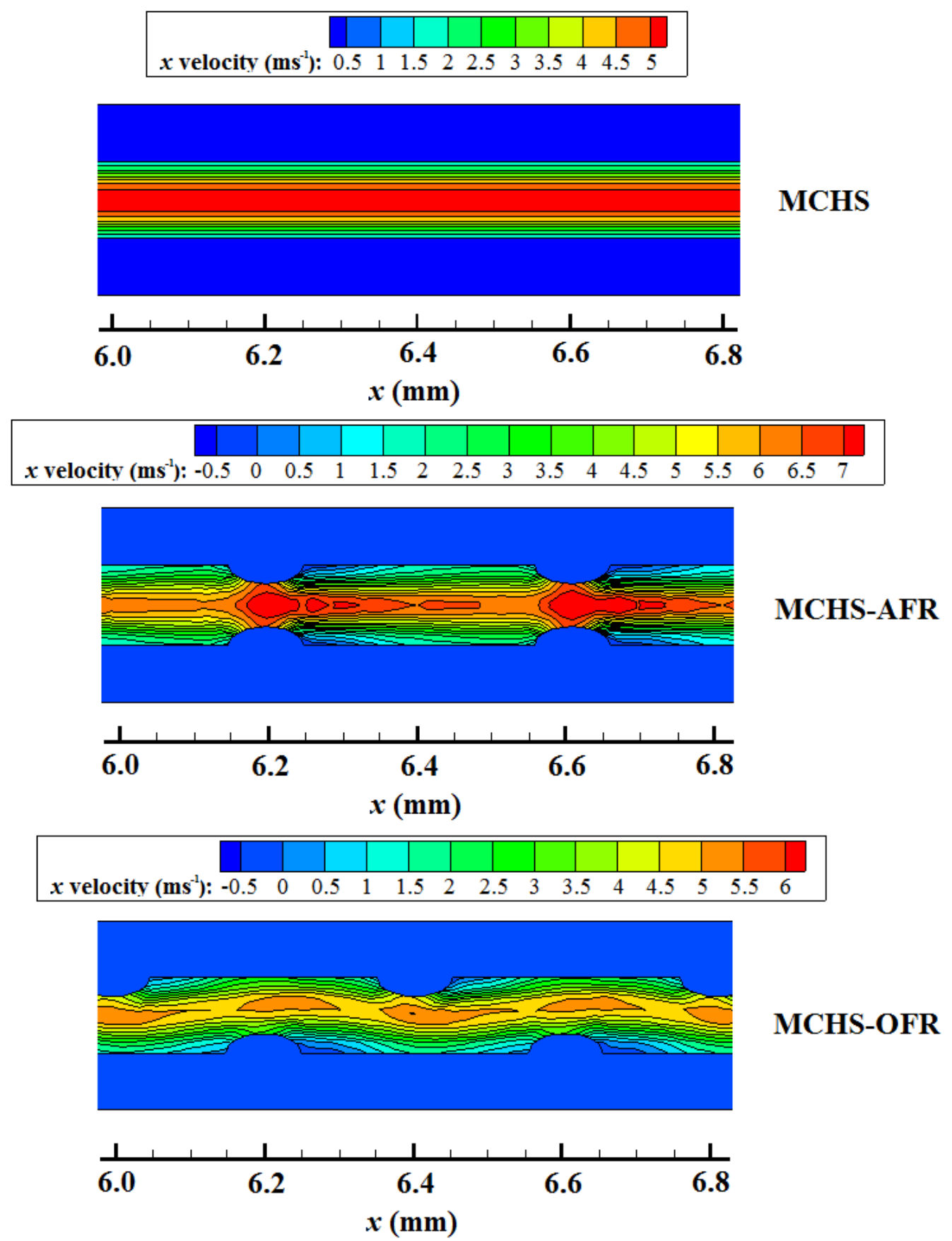

(a) 
Fig. 3b

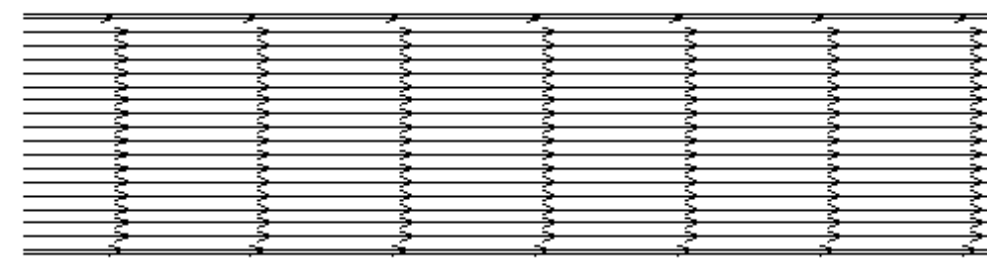

MCHS

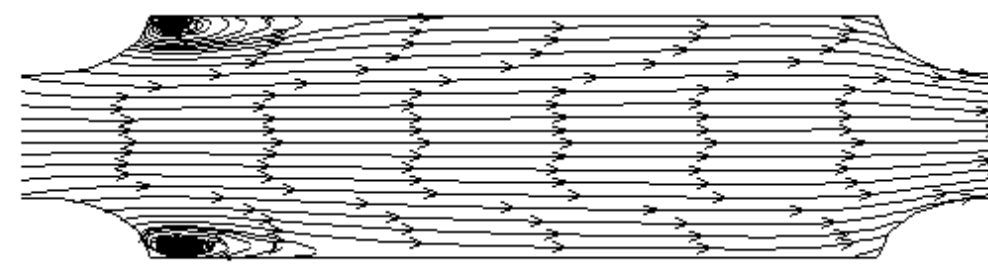

MCHS-AFR

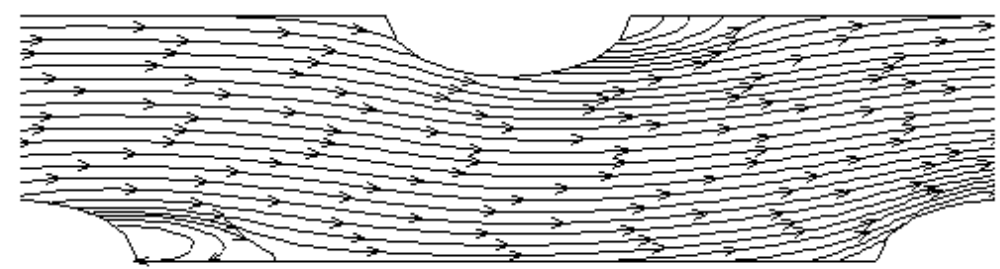

MCHS-OFR

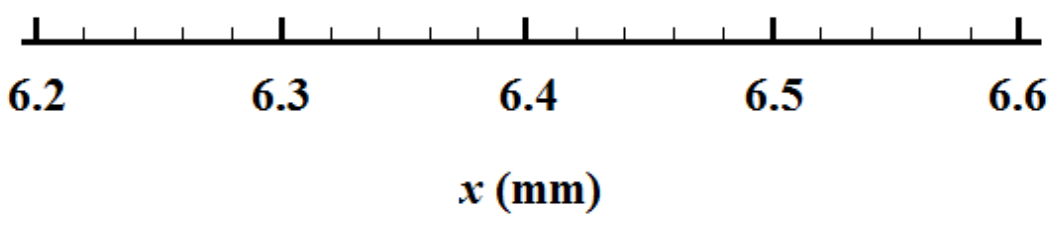

(b) 
Fig. 4
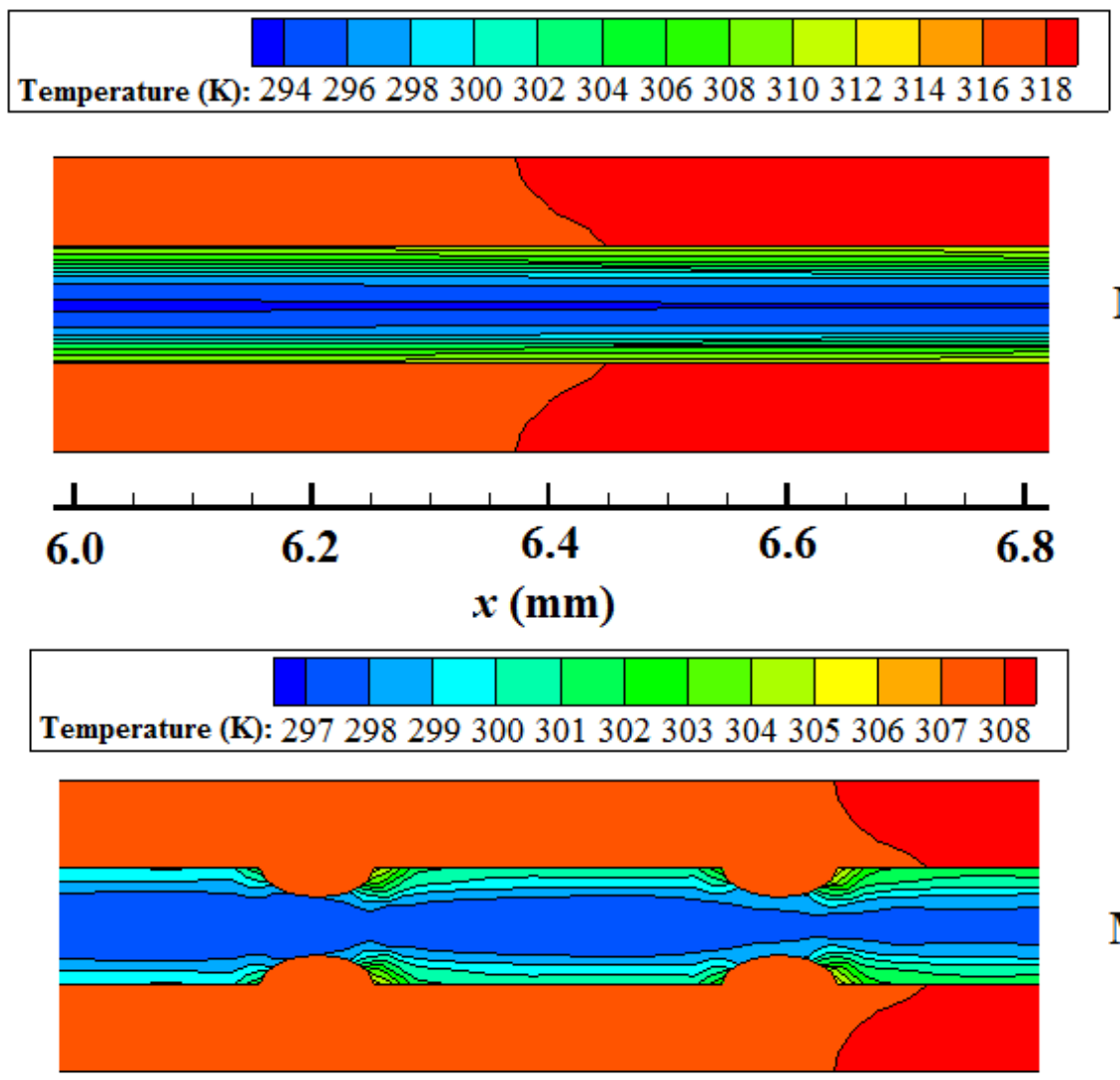

MCHS-AFR

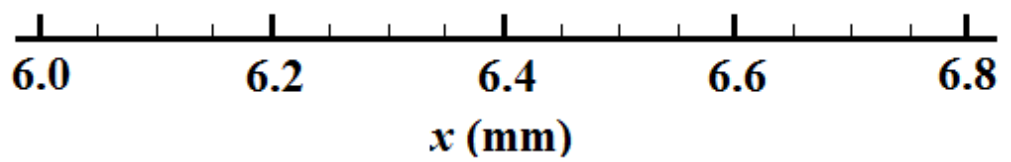

Temperature (K): 297298299300301302303304305306307308

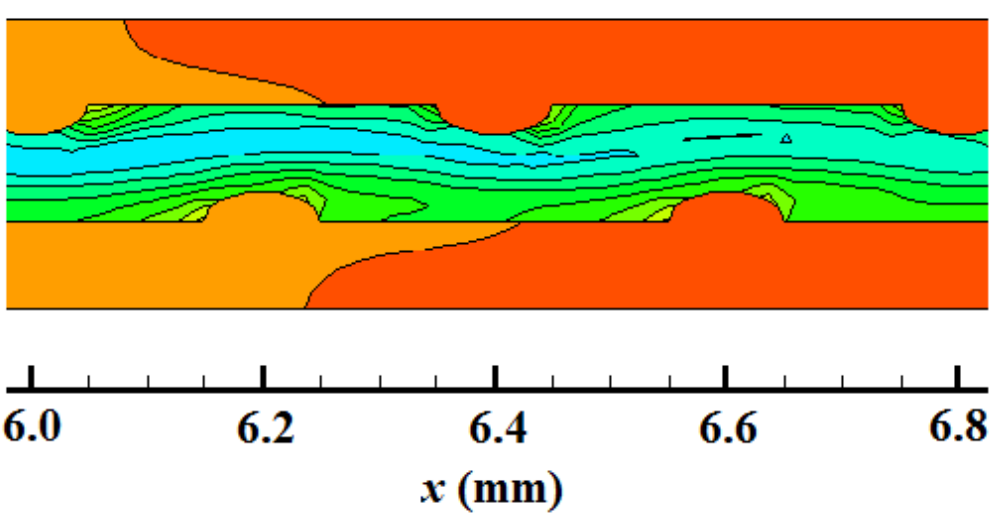

MCHS-OFR 
Fig. 5

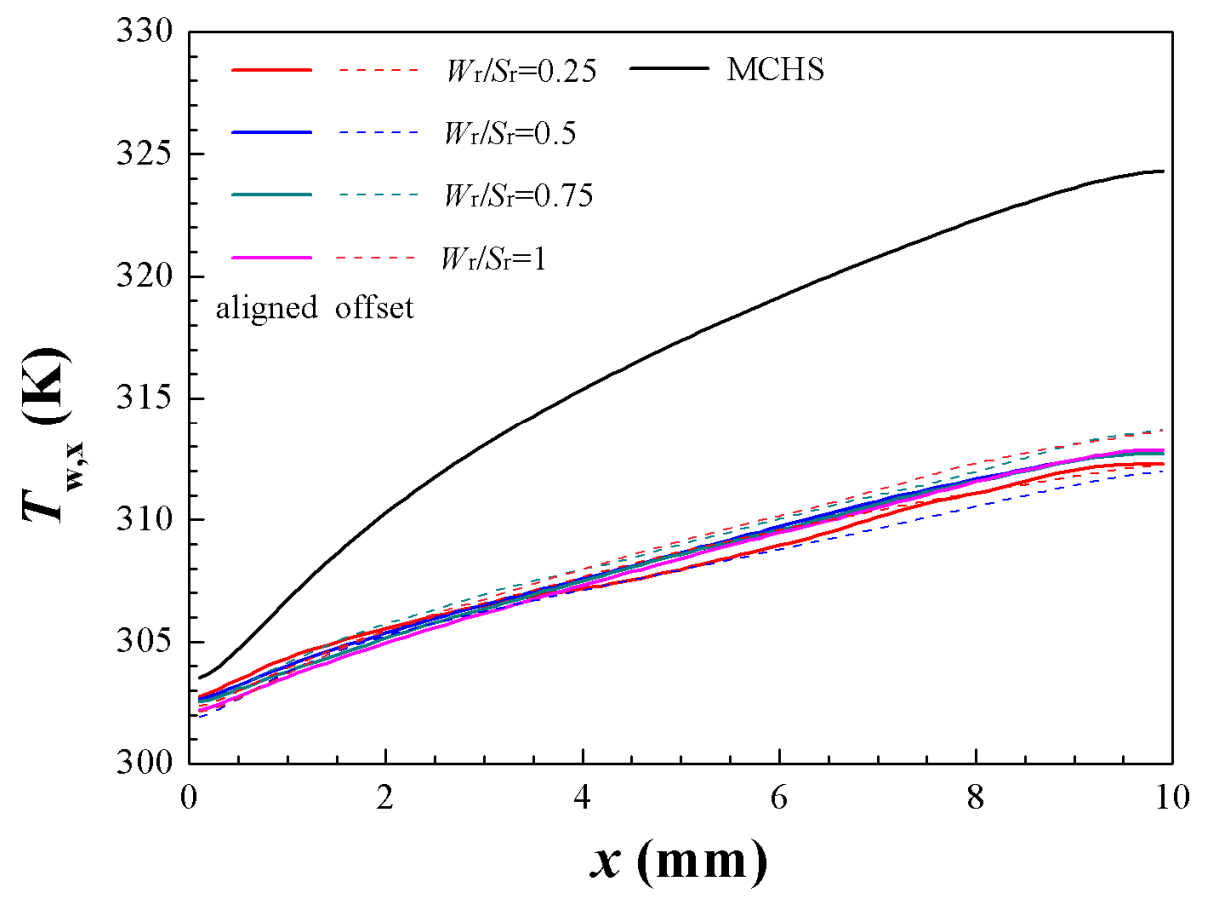

(a)

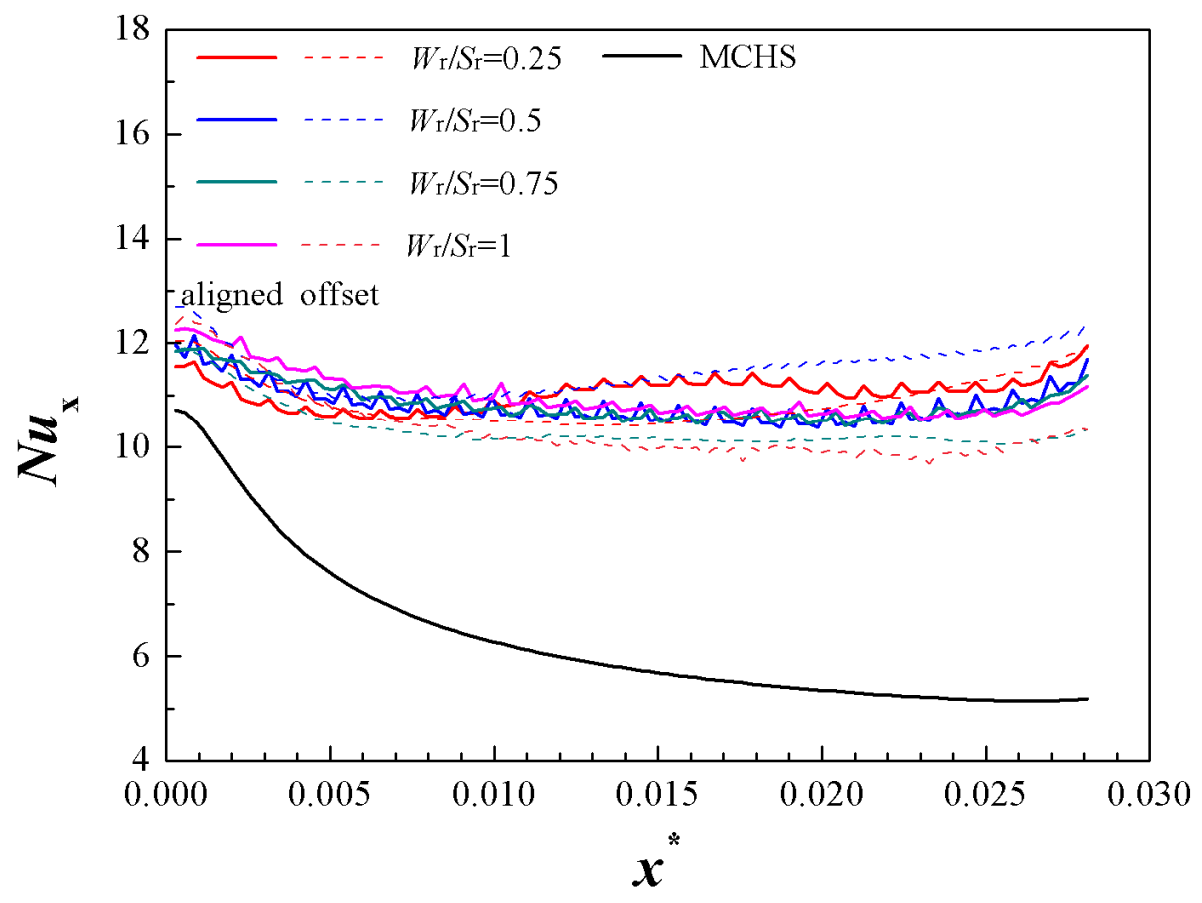

(b) 
Fig. 6

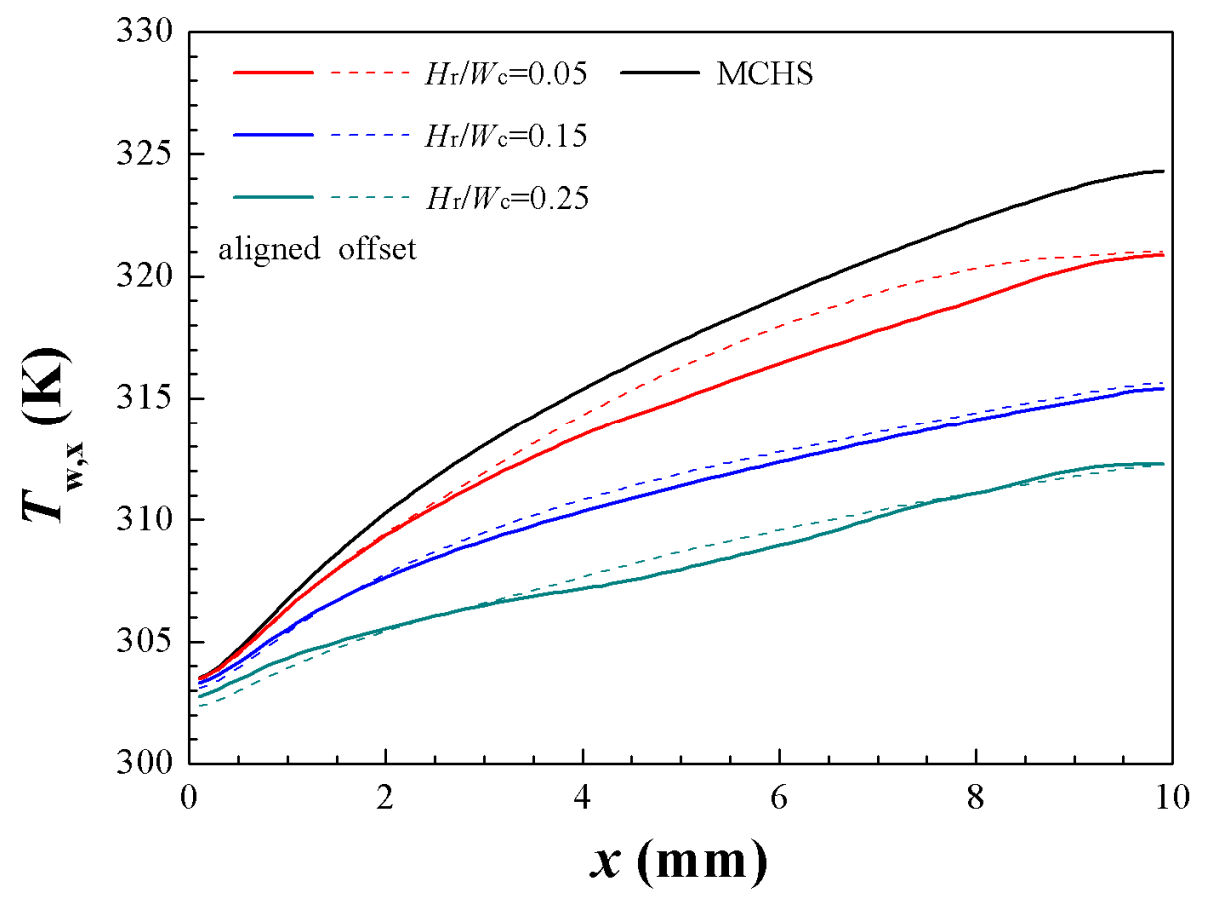

(a)

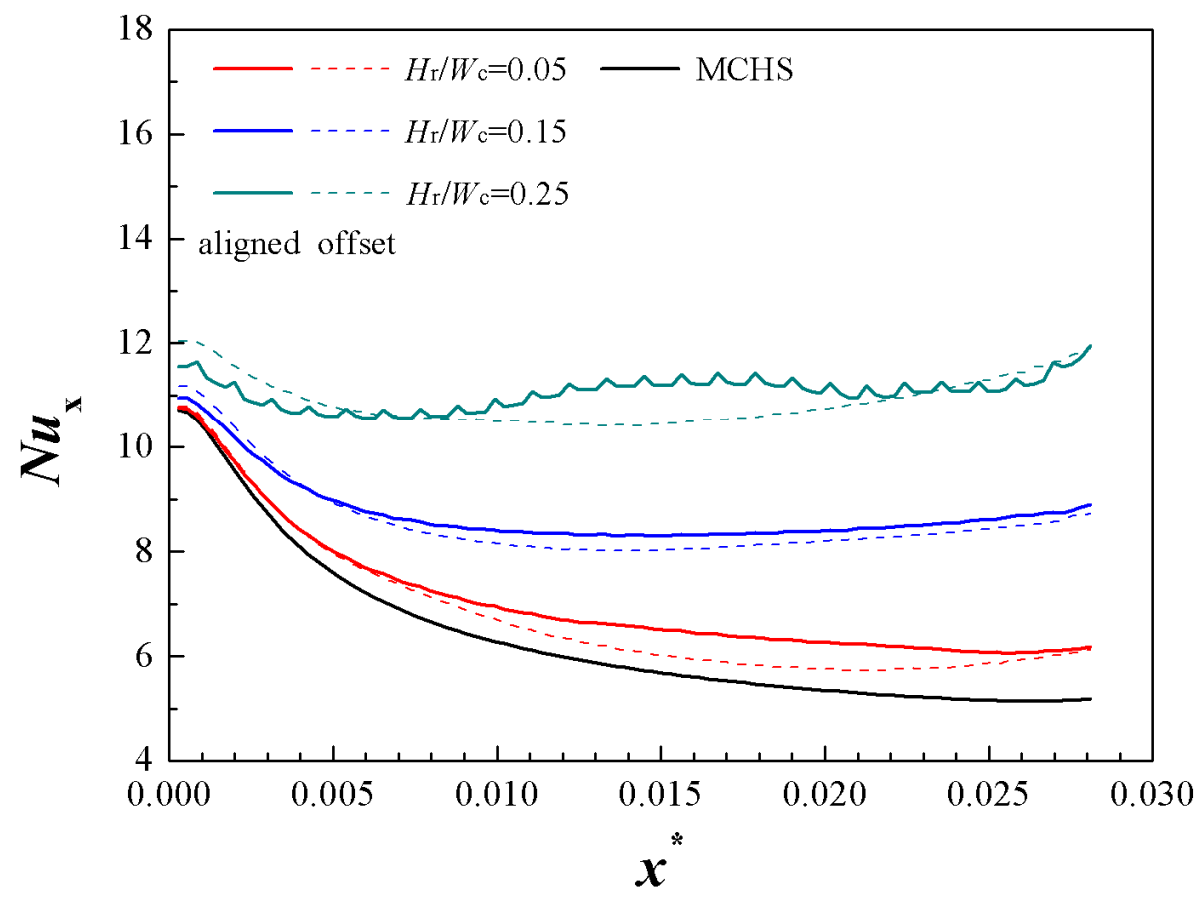

(b) 
Fig. 7

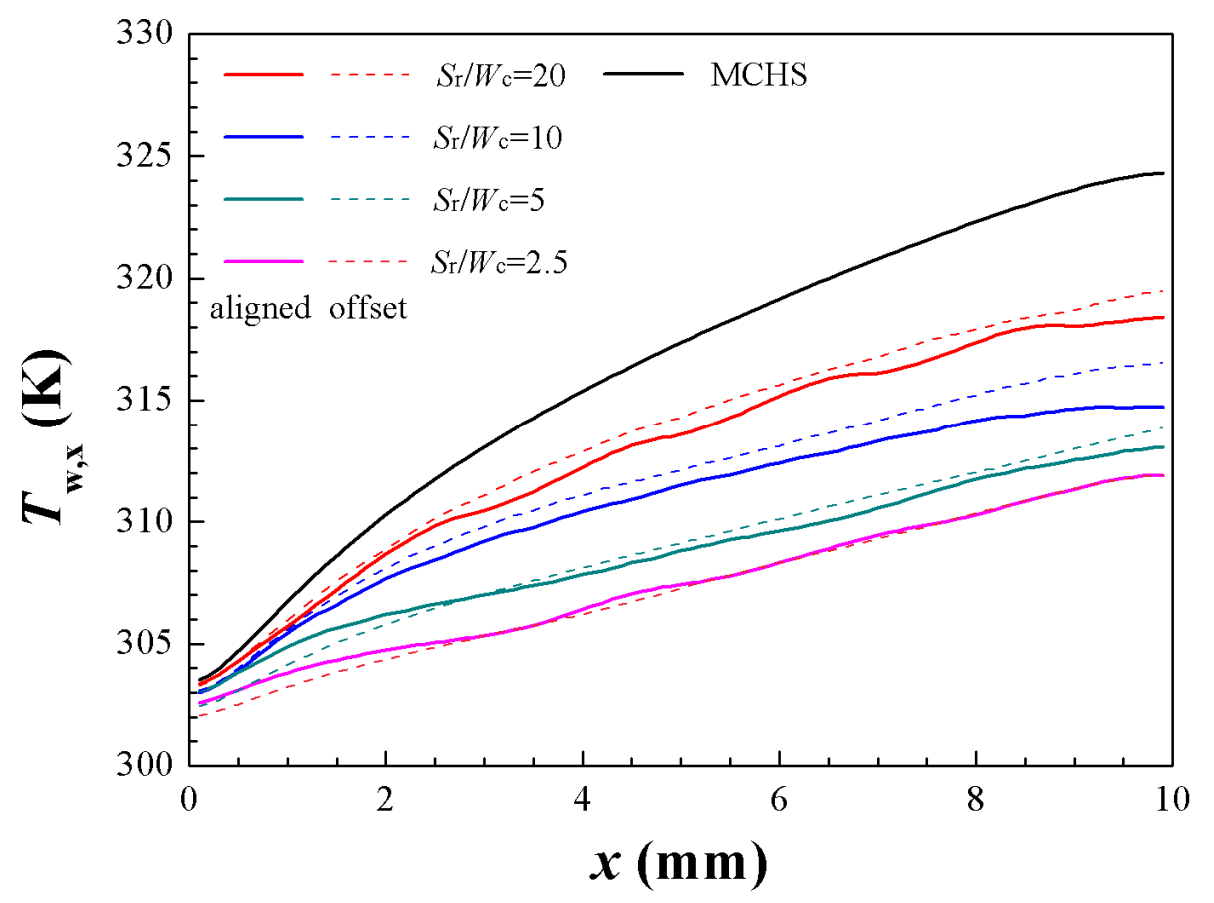

(a)

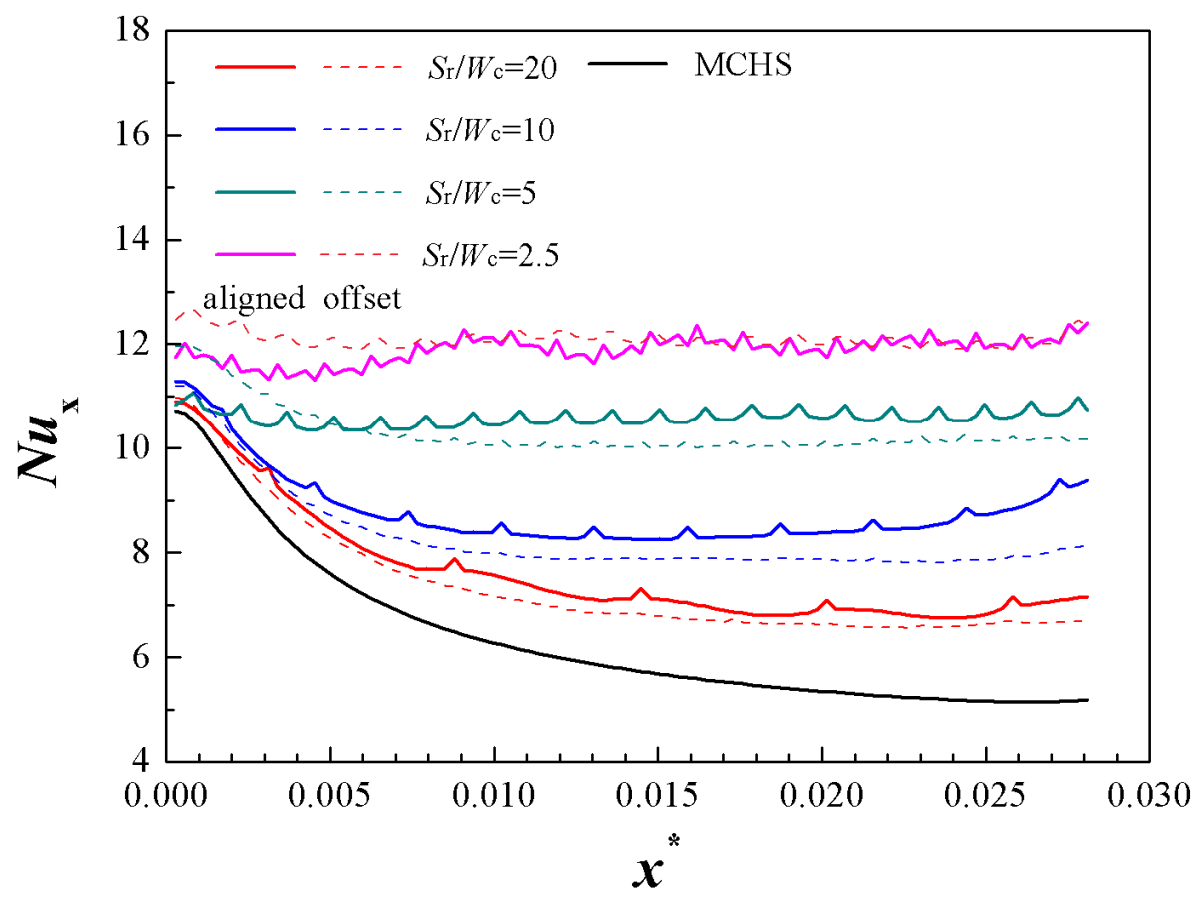

(b) 
Fig. $8 \mathrm{a}$ and $8 \mathrm{~b}$

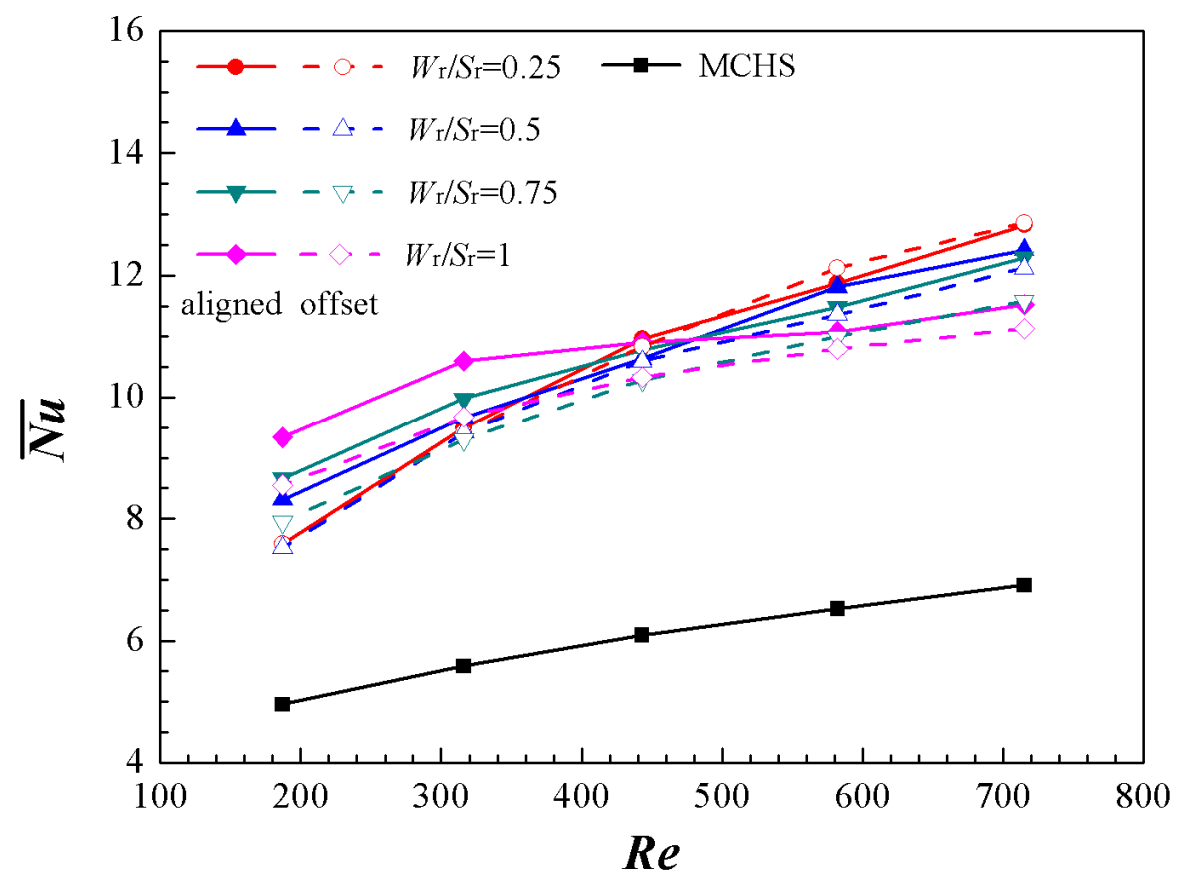

(a)

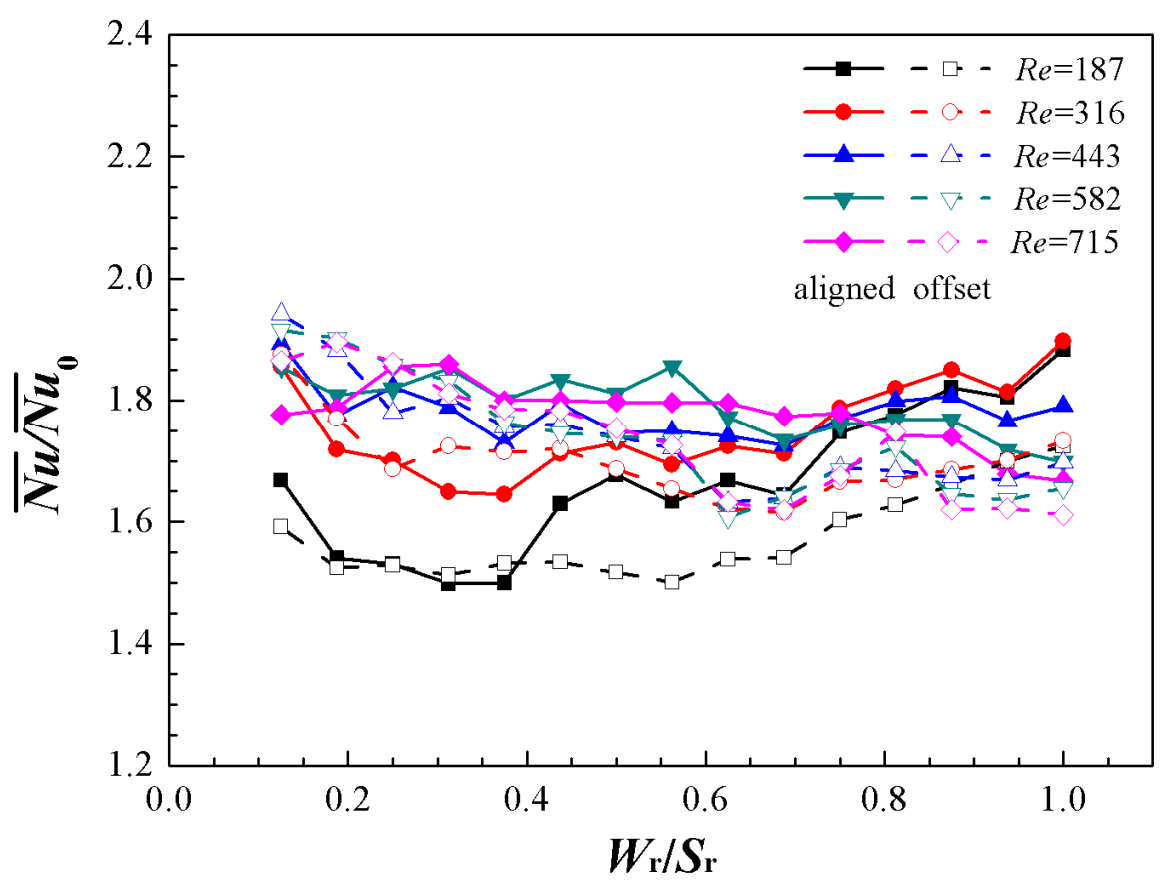

(b) 
Fig. 8c

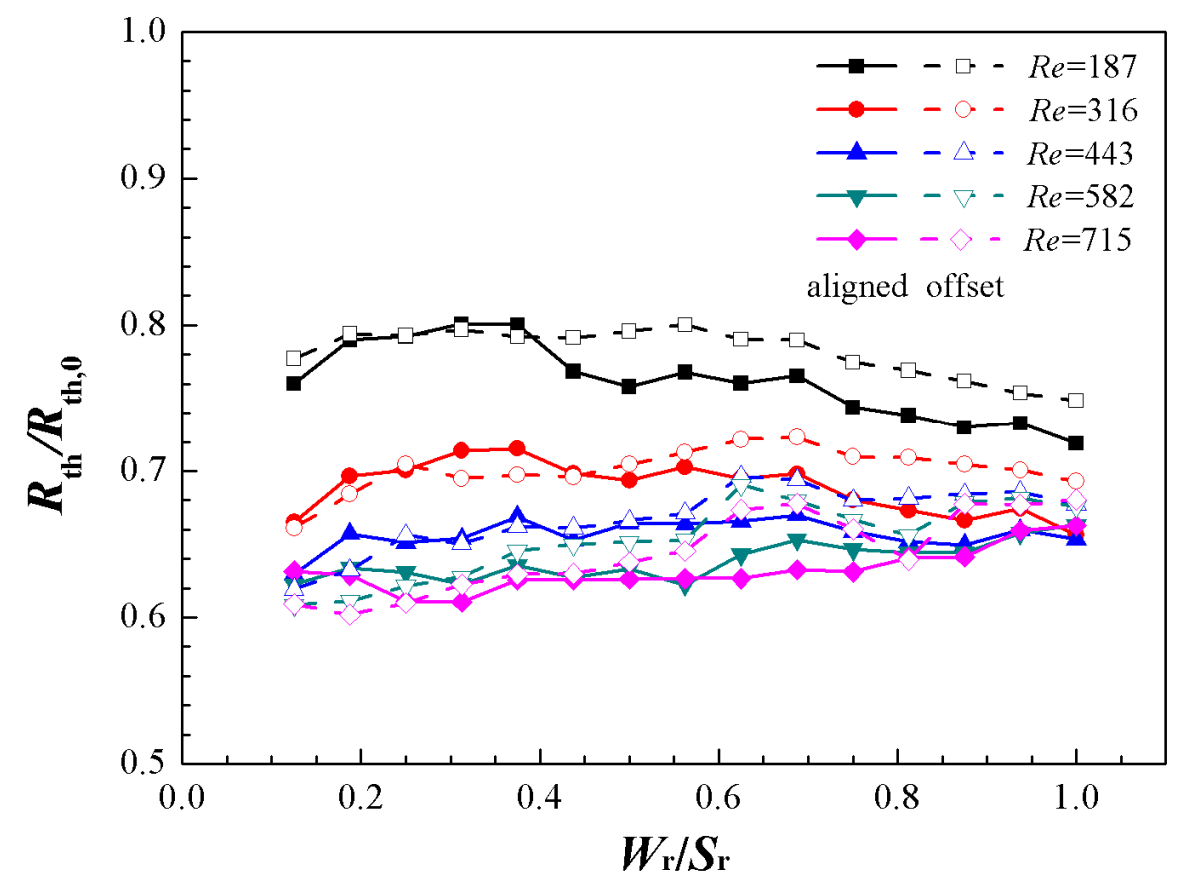

(c) 
Figs. $9 \mathrm{a}$ and $9 \mathrm{~b}$

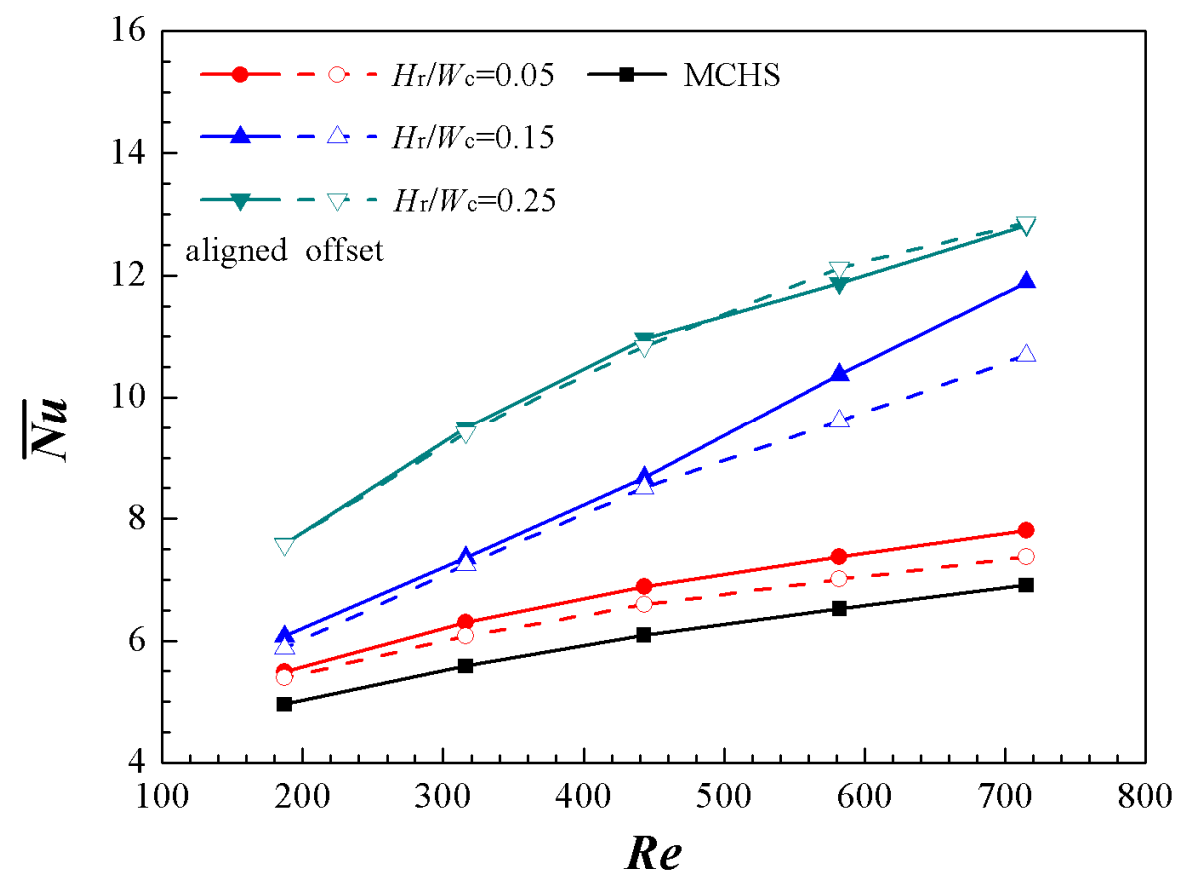

(a)

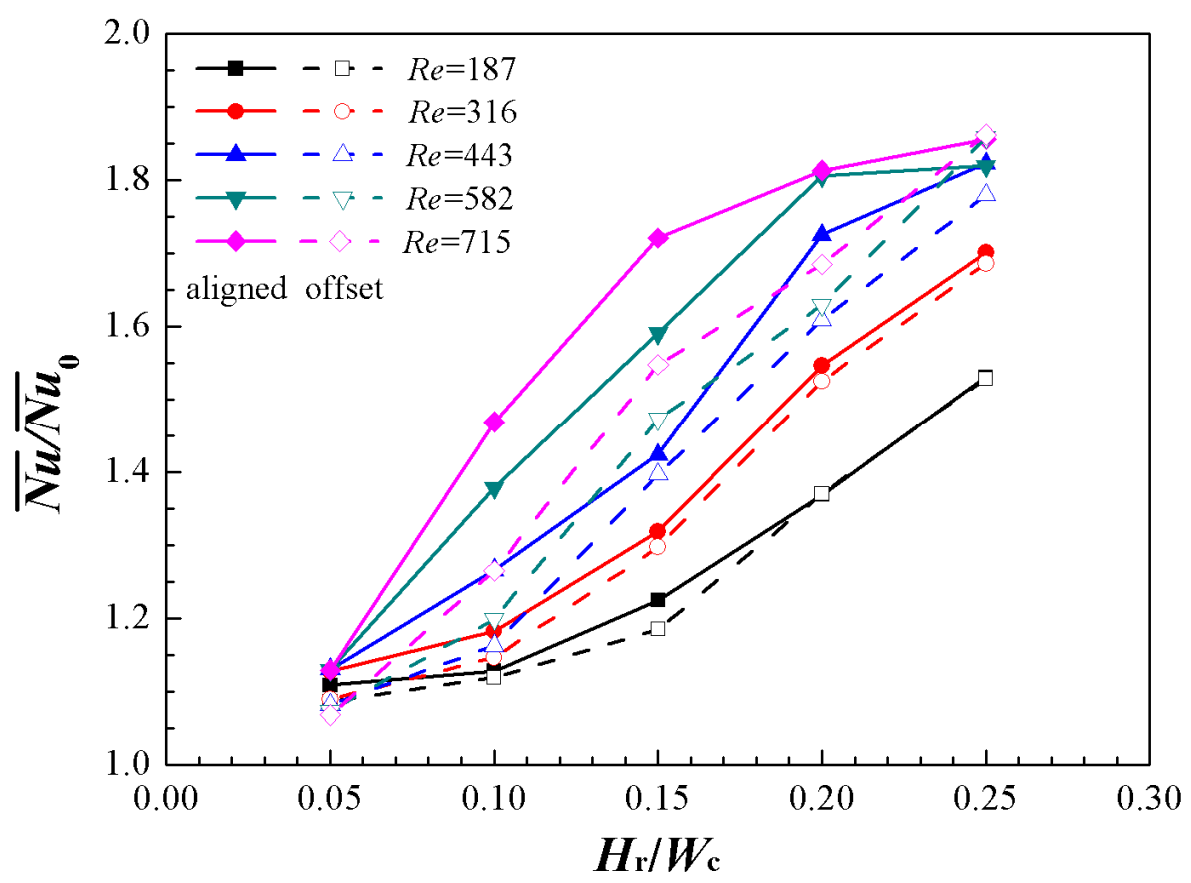

(b) 
Fig. 9c

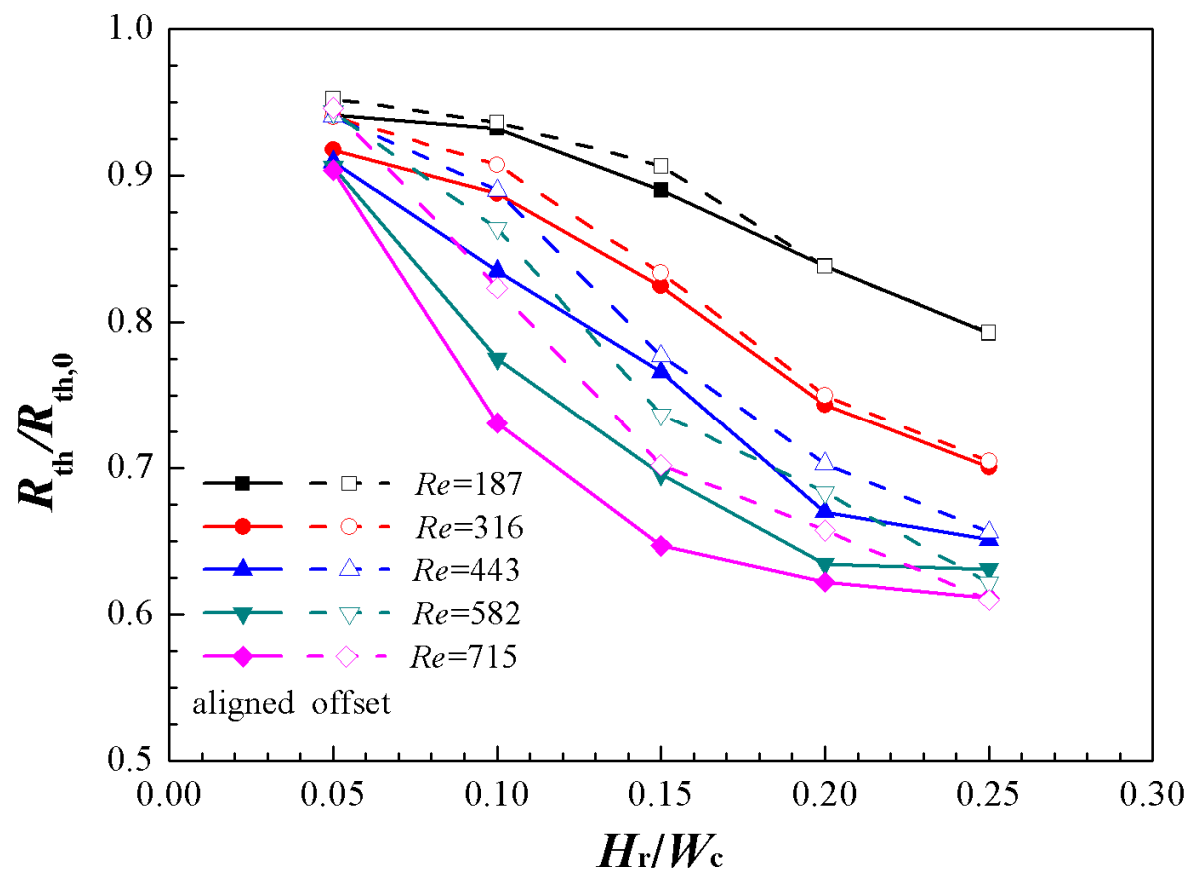

(c) 
Fig. 10a and 10b

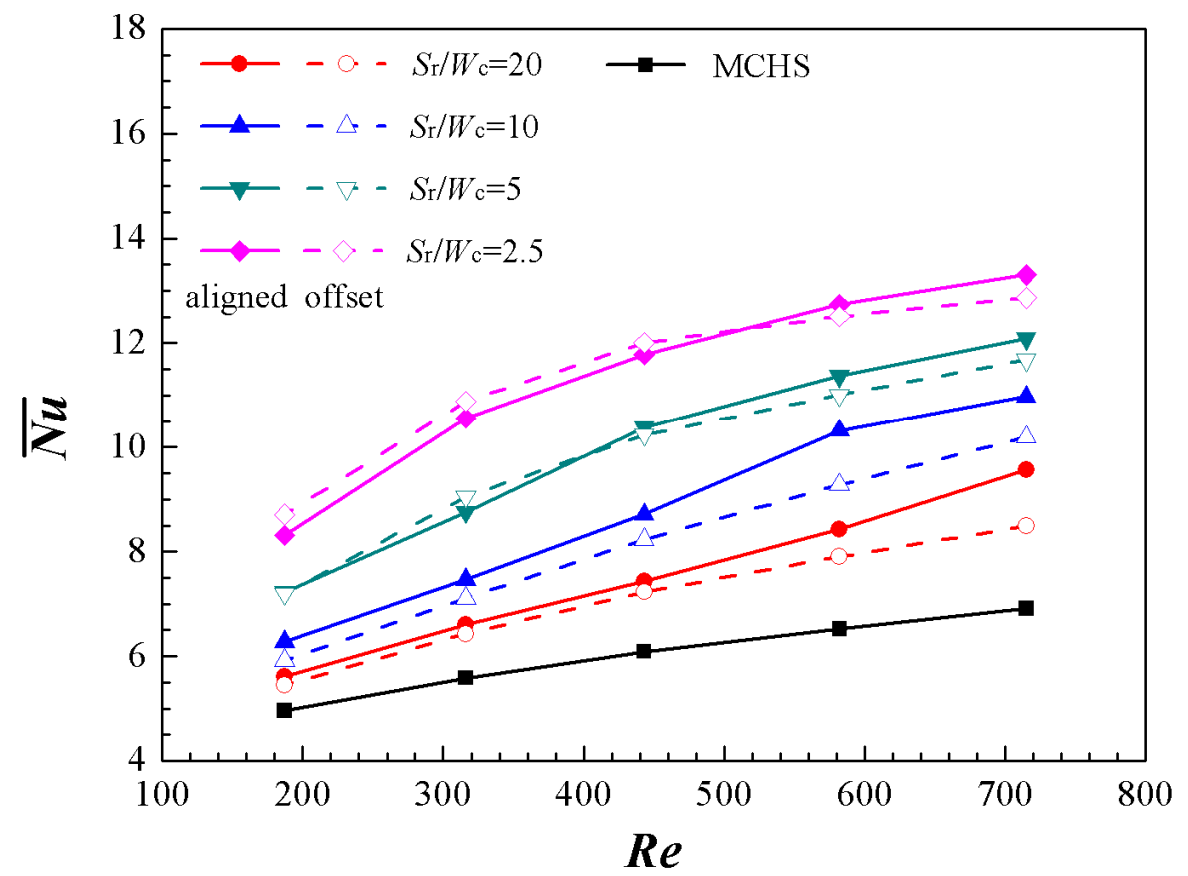

(a)

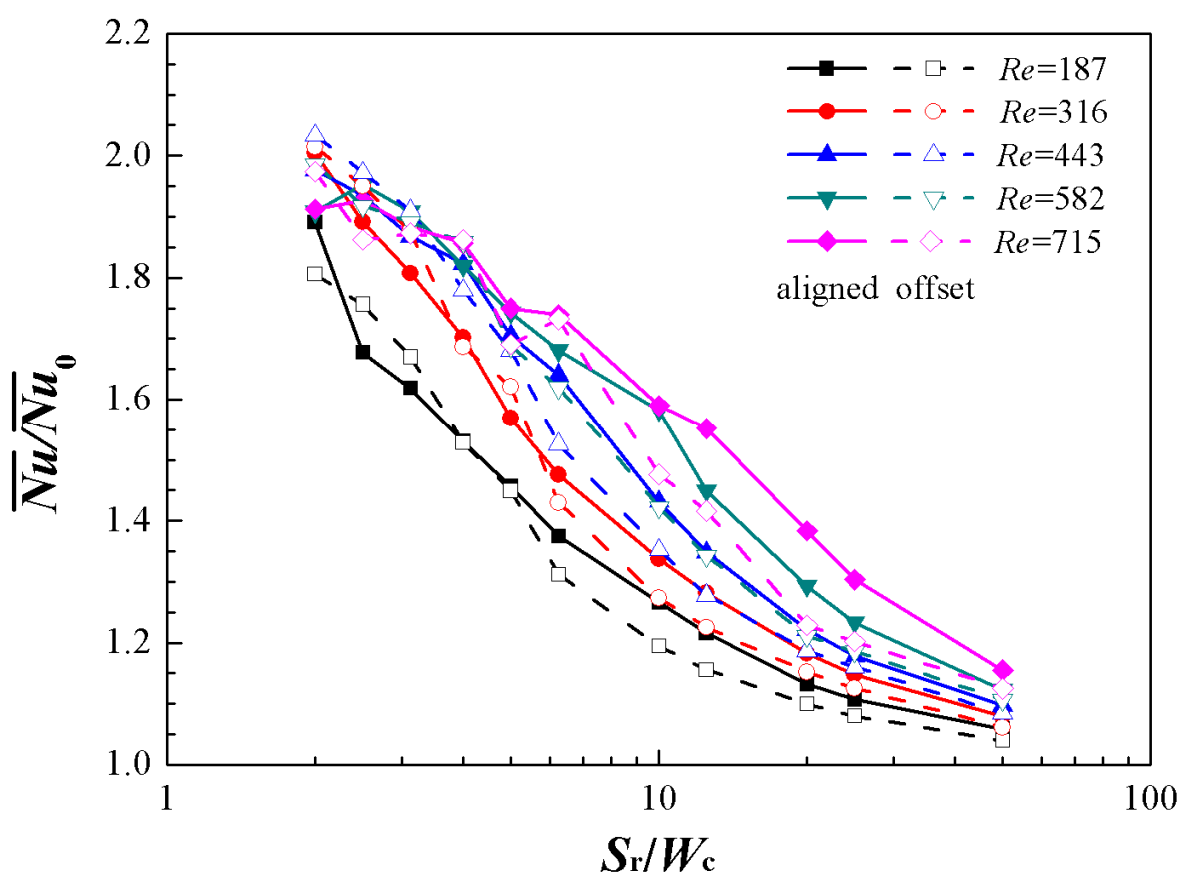

(b) 
Fig. 10c

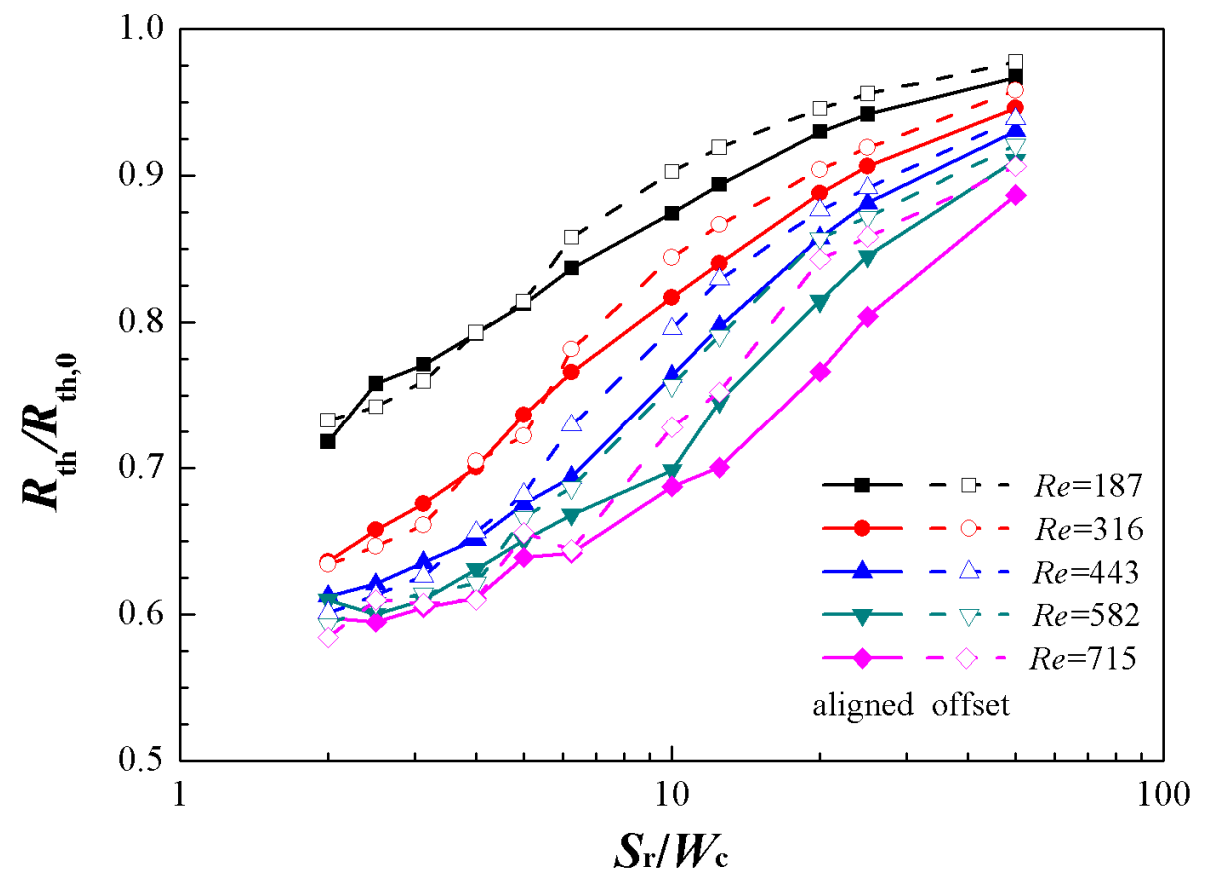

(c) 
Fig. 11

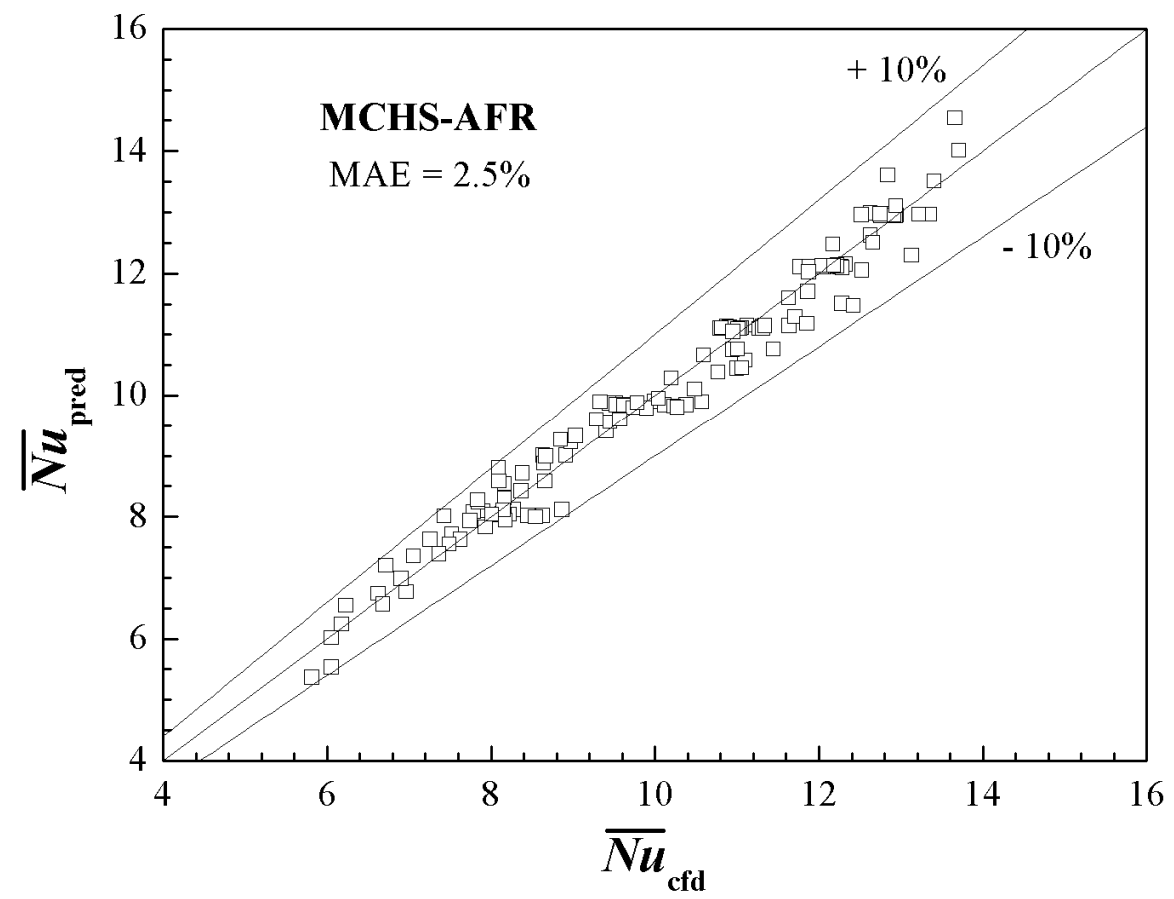

(a)

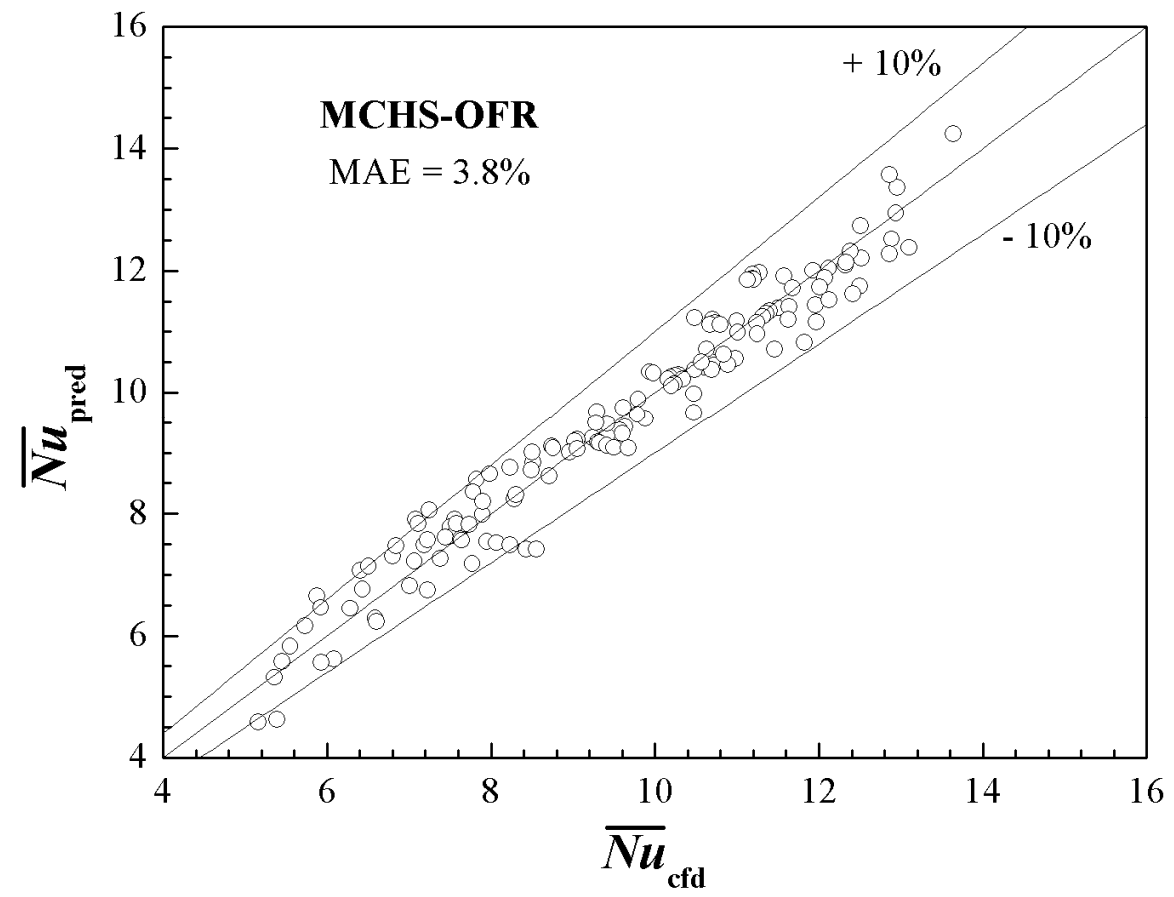

(b) 